\title{
Nearest Prototype Classifier Designs: An Experimental Study
}

\author{
James C. Bezdek, ${ }^{1, *}$ Ludmila I. Kuncheva ${ }^{2,} \dagger$ \\ ${ }^{1}$ Computer Science Department, University of West Florida, Pensacola, Florida \\ 32514 \\ ${ }^{2}$ School of Informatics, University of Wales, LL57 1UT Bangor, United Kingdom
}

We compare eleven methods for finding prototypes upon which to base the nearest prototype classifier. Four methods for prototype selection are discussed: Wilson + Hart (a condensation + error-editing method), and three types of combinatorial search - random search, genetic algorithm, and tabu search. Seven methods for prototype extraction are discussed: unsupervised vector quantization, supervised learning vector quantization (with and without training counters), decision surface mapping, a fuzzy version of vector quantization, $c$-means clustering, and bootstrap editing. These eleven methods can be usefully divided two other ways: by whether they employ pre- or postsupervision; and by whether the number of prototypes found is user-defined or "automatic." Generalization error rates of the 11 methods are estimated on two synthetic and two real data sets. Offering the usual disclaimer that these are just a limited set of experiments, we feel confident in asserting that presupervised, extraction methods offer a better chance for success to the casual user than postsupervised, selection schemes. Finally, our calculations do not suggest that methods which find the "best" number of prototypes "automatically" are superior to methods for which the user simply specifies the number of prototypes. (c) 2001 John Wiley \& Sons, Inc.

\section{INTRODUCTION}

All of the methods discussed here begin with a crisply labeled set of training data $X=\left\{\mathbf{x}_{1}, \ldots, \mathbf{x}_{n}\right\} \subset \mathfrak{R}^{p}$ (the training data). Our presumption is that $X$ contains at least one point with class label $i, 1 \leq i \leq c$. The number of prototypes we are looking for, $|\mathbf{V}|=n_{p}$, depends on the method used, and may be less than, equal to, or greater than $c$, the number of classes represented in $X$.

Let $\mathbf{x}$ be an unlabeled object that we wish to label as belonging to one of $c$ classes. The standard nearest prototype (1-np) classification rule assigns $\mathbf{x}$ to the class of the "most similar" prototype in a set of labeled prototypes (or reference

\footnotetext{
* Author to whom correspondence should be addressed; e-mail: jbezdek@uwf.edu. † e-mail: mas00a@bangor.ac.uk.

Contract grant sponsor: ONR.

Contract grant number: 0014-96-1-0642.
} 
set), say $\mathbf{V}=\left\{\mathbf{v}_{1}, \ldots, \mathbf{v}_{\mathrm{np}}\right\}$. Hereafter, $E_{\mathrm{np}}\left(X_{\mathrm{tr}} ; \mathbf{V}\right)$ denotes the resubstitution (training error) committed by the 1-np rule that uses $\mathbf{V}$ when applied to the training data; $E_{\text {np }}\left(X_{\text {test }} ; \mathbf{V}\right)$ stands for the generalization (testing) error of the same classifier. Why use a nearest prototype classifier? Because it is intuitive, simple, and often, pretty accurate. ${ }^{1}$ Human pattern recognition is almost always based on comparison of the presented input to a catalog of stored examples, both typical and atypical, and this fact is reflected in many case- or instance-based paradigms used in machine learning. ${ }^{2}$

Good prototypes have two desirable properties: minimal cardinality and maximum classification accuracy [or equivalently, minimum $E_{\mathrm{np}}\left(X_{\text {test }} ; \mathbf{V}\right)$ ]. In other words, we seek the smallest possible $\mathbf{V}$ with the highest possible (generalization) accuracy of the 1-np rule that uses V. You expect these two goals to naturally conflict, and our experiments show that they do. Increasing the number of prototypes to some experimentally determined upper limit (usually with $n_{p}>c$ ) almost always results in a decreasing trend in the test error rate $E_{\text {np }}\left(X_{\text {test }} ; \mathbf{V}\right)$, and conversely. One goal of our research is to study this conflict-how to find the smallest set of prototypes that provides an acceptable generalization error for the data at hand.

Various classifiers assign different kinds of labels to unlabeled objects. There are four types of class labels - crisp, fuzzy, probabilistic, and possibilistic. Let $n$ be the number of objects and integer $c$ denote the number of classes, $1 \leq c \leq n$. We define three sets of label vectors in $\mathfrak{R}^{c}$ as follows:

$$
\begin{gathered}
N_{\mathrm{pc}}=\left\{\mathbf{y} \in \mathfrak{R}^{c}: y_{i} \in[0,1] \forall i, y_{i}>0 \exists i\right\}=[0,1]^{c}-\{\mathbf{0}\} \\
N_{\mathrm{fc}}=\left\{\mathbf{y} \in N_{\mathrm{pc}}: \sum_{i=1}^{c} y_{i}=1\right\} \\
N_{\mathrm{hc}}=\left\{\mathbf{y} \in N_{\mathrm{fc}}: y_{i} \in\{0,1\} \forall i\right\}=\left\{\mathbf{e}_{1}, \mathbf{e}_{2}, \ldots, \mathbf{e}_{c}\right\}
\end{gathered}
$$

In Eq. (1) 0 is the zero vector in $\mathfrak{R}^{c} . N_{\mathrm{hc}}$ is the canonical (unit vector) basis of Euclidean $c$-space, so the crisp label for class $i, 1 \leq i \leq c$, is

$$
\mathbf{e}_{i}=(0,0, \ldots, \underbrace{1}_{i}, \ldots, 0)^{T},
$$

the $i$ th vertex of $N_{\mathrm{hc}}$. The set $N_{\mathrm{fc}}$, a piece of a hyperplane, is the convex hull of $N_{\text {hc }}$. The vector $\mathbf{y}=(0.1,0.6,0.3)^{\mathrm{T}}$ is a constrained label vector; its entries lie between 0 and 1 , and sum to 1 . The centroid of $N_{\mathrm{fc}}$ is the equimembership vector $\mathbf{1} / \mathbf{c}=(1 / c, \ldots, 1 / c)^{T}$. If $\mathbf{y}$ is a label vector for some $\mathbf{x} \in \Re^{p}$ generated by, say, the fuzzy $c$-means clustering method, we call $\mathbf{y}$ a fuzzy label for $\mathbf{x}$. If $\mathbf{y}$ came from a method such as maximum likelihood estimation in mixture decomposition, $\mathbf{y}$ would be a probabilistic label. In this case, $\mathbf{1 / c}$ is the unique point of equal probabilities for all $c$ classes.

$N_{\mathrm{pc}}=[0,1]^{c}-\{\mathbf{0}\}$ is the unit hypercube in $\mathfrak{R}^{c}$, excluding the origin. Vectors, such as $\mathbf{z}=(0.7,0.2,0.7)^{T}$ with each entry between 0 and 1 that are otherwise 
unrestricted, are possibilistic labels in $N_{p 3}$. We interpret possibilistic label values as indicants of the extent to which $\mathbf{z}$ is typical of each of the $c$ classes. Possibilistic labels are produced by possibilistic clustering algorithms ${ }^{3}$ and by computational neural networks that have unipolar sigmoidal transfer functions at each of $c$ output nodes. ${ }^{4}$ For convenience we call all noncrisp labels soft labels. Note that $N_{\text {hc }} \subset N_{\text {fc }} \subset N_{\text {pc }}$.

In Section II we discuss prototype extraction and the generalized nearest prototype classifier (GNPC). ${ }^{5,6}$ Section III contains a concise description of the four selection methods surveyed in this article, and Section IV describes the seven replacement methods we chose to compare to the four selection methods. The four data sets used and our results - comparisons between, and similarities of, the eleven methods-are discussed in Section V. Section VI offers the conclusions we draw from these experiments, and itemizes some interesting topics for further research.

\section{PROTOTYPE EXTRACTION AND THE GNPC}

When the prototypes used in the GNPC have soft labels, each prototype may "vote" with varying assurance for all $c$ classes. For example, if $\mathbf{v}_{i}$ has the soft label [0.2, 0.4,0.7], this prototype is a fairly typical example of class 3 but is also related (less strongly) to classes 1 and 2. A real-life example of soft labeling is diagnosing ischemic heart disease, where the occlusion of the three main coronary arteries can be expressed by such a label, each entry being the degree of occlusion of a particular vessel.

If $\mathbf{x}$ and the $\mathbf{v}_{i} \mathbf{s}$ are represented by feature vectors in $\Re^{p}$, prototypical similarity is almost always based on some function of pairwise distances between $\mathbf{x}$ and the $\mathbf{v}_{i}$ s. Specifically, let $\mathbf{x} \in \mathfrak{R}^{p}$ be an input vector. The GNPC is defined by the 5-tuple:

(1) The set of prototypes $\mathbf{V}=\left\{\mathbf{v}_{1}, \ldots, \mathbf{v}_{n_{p}}\right\} \subset \Re^{p}$.

(GNPC1)

(2) The $c \times n_{p}$ prototype label matrix $L(\mathbf{V})=\left[\ell\left(\mathbf{v}_{1}\right), \ldots, \ell\left(\mathbf{v}_{n_{p}}\right)\right] \in \Re^{c} \times \Re^{n_{p}}$.

(GNPC2)

(3) A similarity function $S\left(\mathbf{x}_{k}, \mathbf{v}_{i}\right)=\Theta\left(\left\|\mathbf{x}_{k}-\mathbf{v}_{i}\right\|\right)$ valued in [0,1] that assesses the similarity between inputs and prototypes (the higher the value, the more similar the input to the prototype).

(GNPC3)

(4) A $T$-norm (in the fuzzy sets sense) to fuse each prototype label and the similarity between that prototype and $\mathbf{x}$.

(GNPC4)

(5) An aggregation operator which uses the $p$ fused components to produce an overall soft label for $\mathbf{x}$.

(GNPC5)

When the output of (GNPC5) is a soft label, a crisp class label can always be assigned to $\mathbf{x}$ by taking the class with the highest "support" in a soft label vector. This operation is a specific form of the general operation of hardening soft labels. A great number of classifier models can be described by different choices of the parameters in (GNPC1-GNPC5), and an even greater set can be designed by varying those parameters. References 5 and 6 contain detailed descriptions and many examples of models that fall into the GNPC framework. 
Figure 1 shows three of the many groups of classifiers that belong to the GNPC family. Abbreviations appearing in Figure 1 are: hard c-means (HCM), nearest neighbor (1-nn), learning vector quantization (LVQ), and radial basis function (RBF). Many of the classifiers in Figure 1 are simple 1-np classifiers; the major distinction between them is in the way their prototypes are obtained. This paper discusses some cases of the models shown in the module highlighted by a heavy border at the bottom left in Figure 1. Three basic characteristics of prototype extraction methods, itemized here as (C1), (C2), and (C3), are discussed next.

(C1) Selection $\left(\mathbf{V}_{\mathrm{s}} \subseteq X\right)$ versus replacement $\left(\mathbf{V}_{\mathrm{r}} \not \subset X\right)$. When talking about prototypes in general, we use the symbol $\mathbf{V}$; when emphasis on selection or replacement is needed, we use subscripts $\left(\mathbf{V}_{s}\right.$ for selection, $\mathbf{V}_{r}$ for replacement). Replacement seeks $n_{p}$ points in $\Re^{p}$, so the search space is infinite. Selection is limited to searching within $X \subseteq \Re^{p}$, so solutions in this case can be sought by combinatorial optimization. Selection is, of course, a special case of prototype replacement. Figure 2 illustrates the two styles of prototype extraction.

(C2) Presupervised versus postsupervised designs. ${ }^{7}$ Presupervised methods use the data and the class labels to locate the prototypes. Postsupervised methods first find prototypes without regard to the training data labels, and then assign a class label (relabel) to each prototype. Selection methods are naturally presupervised because each prototype is a data point and already has its (presumably true) label.

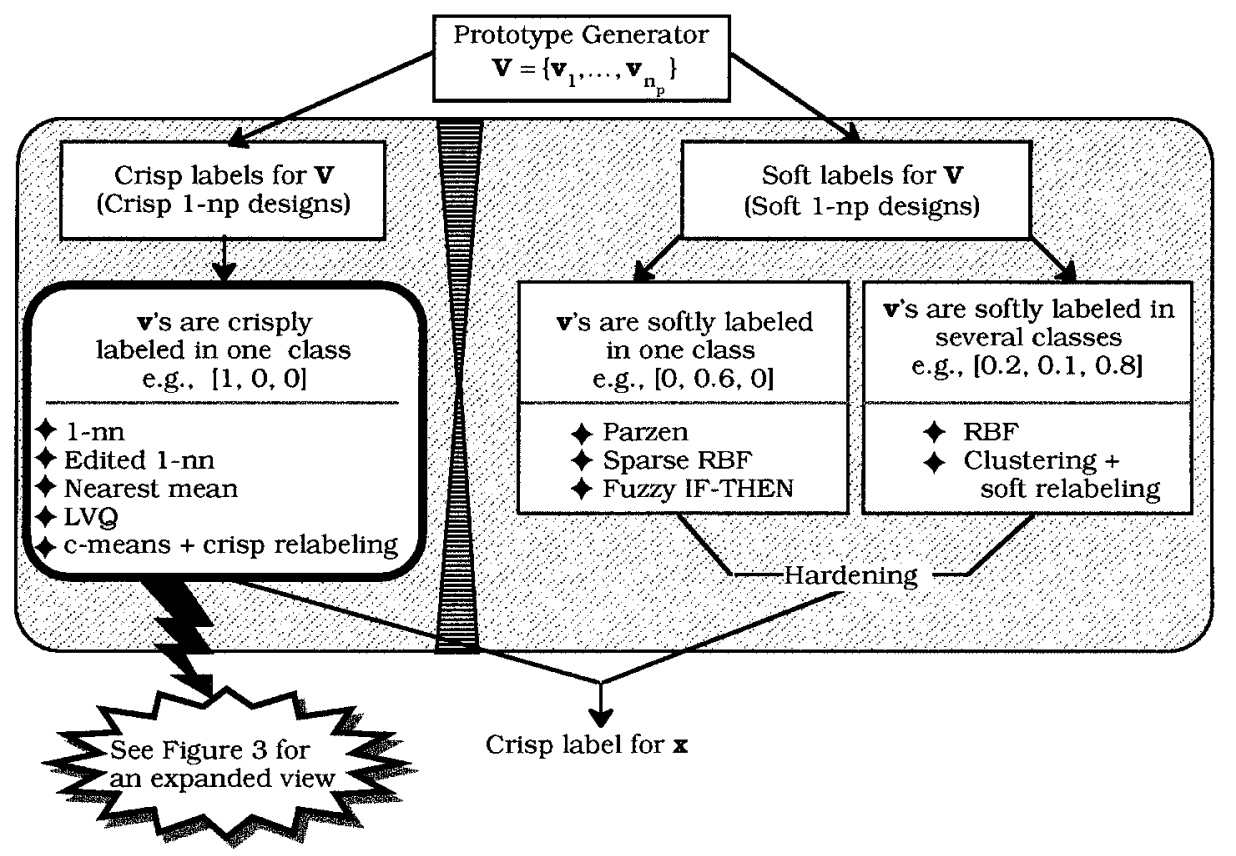

Figure 1. A few models that are generalized nearest prototype classifiers. 

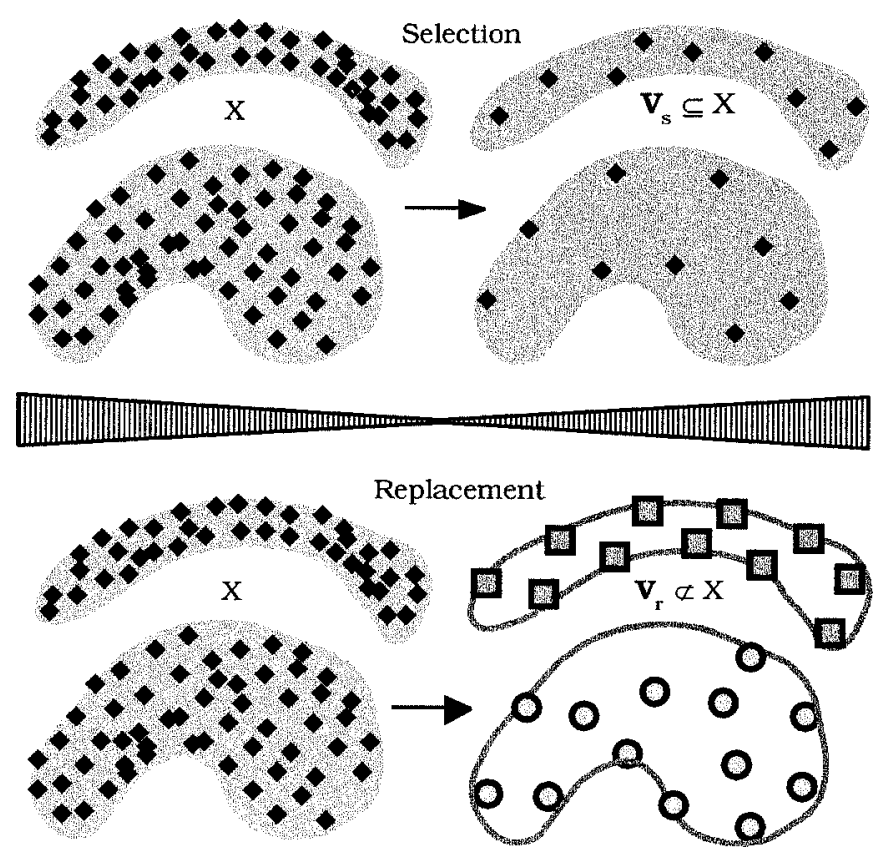

Figure 2. Prototype extraction: selection vs. replacement.

(C3) User-defined $n_{p}$ versus algorithmically defined $n_{p}$. Most methods for prototype generation require $n_{p}$ to be specified in advance as an explicit parameter of the algorithm (e.g., classical clustering and competitive learning methods). Some models have "adaptive" variants where the initially specified value of $n_{p}$ can increase or decrease, i.e., prototypes are added or deleted during training under the guidance of some mathematical criterion of prototype "quality." A third group of methods do not specify $n_{p}$ at all, instead obtaining it as an output at the termination of training. For example, condensation methods which search for a minimal possible consistent set belong to this category. Genetic algorithms and tabu search methods have a trade-off parameter which pits the weight of a misclassification against an increase in the cardinality of $\mathbf{V}$ by 1 . Thus, methods based on these types of search deliver the number of prototypes at termination of training. A method that finds $n_{p}$ during training will be called an auto- $n_{p}$ method; otherwise, the method is $u$ ser- $n_{p}$.

Issue (C3) is an often misunderstood aspect of prototype generation for classifier design. The "user-friendliness" of a nearest prototype classifier design is greatly increased by any algorithm that "automatically" finds the best number of prototypes, thereby relieving the designer of making a tradeoff study to select $n_{p}$. Do auto- $n_{p}$ methods really find "better" prototypes than user- $n_{p}$ methods? Of course not, but there is one less parameter for the user to pick, so auto- $n_{p}$ methods enjoy popularity on this account (and, we can call them "adaptive"!). 


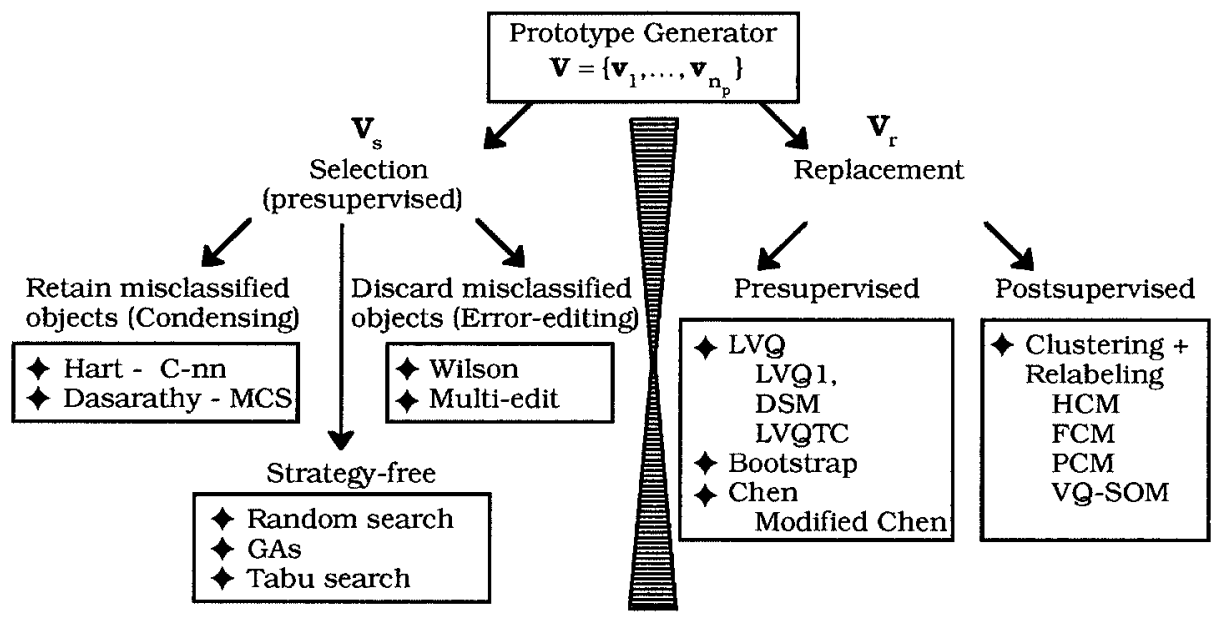

Figure 3. Methods for finding prototypes.

An expanded view of the highlighted module in the lower left corner of Figure 1 is shown in Figure 3. New abbreviations appearing in Figure 1 are: fuzzy c-means (FCM), possibilistic c-means (PCM), condensed nearest neighbor (C-nn), self organizing map (SOM), decision surface mapping (DSM), minimal consistent subset (MCS), LVQ (with) Training Counters (LVQTC), and genetic algorithm (GA). The left half of Figure 3 shows prototype selection divided into two main groups: Condensing (or condensation) and Error-editing (or simply "editing").

Prototype replacement is also divided into two main groups in Figure 3: presupervised and postsupervised methods. The framework provided by the GNPC model gives us a common platform from which to view many of these methods from the same perspective. For example, we see here both VQ and LVQ, along with some of the many modifications thereof. ${ }^{8-12}$ DSM was introduced by Geva and Sitte. ${ }^{13}$ Akin to the VQ family is the clustering-and-relabeling approach which itself has many variations, the most popular of which are perhaps the $c$-means families shown in Figure $3 .{ }^{1}$ Cluster centroids are used as the replacement prototypes $\mathbf{V}_{r}$, so any clustering method that produces centroids can be used for this purpose. Having overviewed a piece of the GNPC forest, we turn to a closer look at a few of its trees.

\section{PROTOTYPE SELECTION}

In this section we give brief overviews and the computational protocols for the four selection methods used in our numerical experiments. Following the architecture of Figure 3, we have subsections for condensation, error-edition, 
and search methods. For convenience in Sections III and IV, we drop the subscript of $X_{\mathrm{tr}}$, and refer to the training data more simply as $X$.

\section{A. Selection by Condensation}

The objective of condensation is to find a consistent reference set; i.e., a set $\mathbf{V}_{s} \subset X$, such that $E_{\mathrm{np}}\left(X_{\mathrm{tr}} ; \mathbf{V}_{s}\right)=0$, i.e., the resubstitution error rate of the 1-np classifier is zero. If there are no identical points with different class labels in $X$, then at worst $X$ is a consistent set of $n$ prototypes for itself. Condensation aims to find $\mathbf{V}_{s}$ with the minimal possible cardinality, ideally with $n_{p} \ll n$. This leads to retaining in $\mathbf{V}_{s}$ points from $X$ that are close to classification boundaries. Thus, boundaries, together with all the noise that the boundary data points might involve, are modeled as precisely as possible.

Condensation only guarantees zero resubstitution error. The amount of reduction of the reference set and the generalization capability of the nearest prototype classifier built with the selected $\mathbf{V}_{s}$ are not explicitly involved in condensation selection strategies. However, the general goal of most methods for finding prototypes is to be able to use $\mathbf{V}_{s}$ with some form of the GNPC to label unknown objects. Then generalization error becomes an important issue. A severe drawback of all condensation methods is that the zero resubstitution error requirement is always enforced, so no tradeoff between error rate and the cardinality of $\mathbf{V}_{s}$ is possible.

Hart's C-nn: The original condensation method is the condensed nearest neighbor (C-nn) method of Hart. ${ }^{14}$ Hart's C-nn operates with two sets: STORE and GRABBAG. Initially STORE is empty and all elements of $X$ are placed in GRABBAG. The first element of $X$ is moved from GRABBAG into STORE. The next element is classified using the nearest prototype classifier and the current content of STORE. If misclassified, that element is moved from GRABBAG to STORE. When all elements of GRABBAG have been checked, a new iteration starts at the beginning of GRABBAG, using the current content of STORE. The procedure stops when a pass through GRABBAG has not added any element to STORE (or when GRABBAG is empty, and STORE is $X$ ). The final content of STORE is taken as $\mathbf{V}_{s}$. Many modifications of, and algorithms similar to, Hart's C-nn are known. ${ }^{15}$

The output of Hart's C-nn depends on the order of presentation of the elements in $X$. Different permutations of $X$ can lead to different $\mathbf{V}_{s}$ s. Cerveron and Ferri ${ }^{16}$ suggest running the C-nn multiple times, beginning with different permutations of $X$. Since C-nn builds STORE gradually, as soon as STORE in the current run reaches the cardinality of the smallest $\mathbf{V}_{s}$ found so far, the run is terminated and a new run is started from a different permutation of $X$. This speeds the algorithm towards its destination and seems to produce good sets of consistent prototypes. A minimal consistent set algorithm (MCS) for condensation was proposed by Dasarathy. ${ }^{17}$ Dasarathy's MCS decides which elements to retain after a pass through all of $X$, so unlike C-nn, MCS does not depend on the order in which the elements of $X$ are processed. MCS, however, does not necessarily find $\mathbf{V}_{s}$ with the true minimal cardinality. ${ }^{6}$ 


\section{B. Selection by Editing}

Wilson's Method: Error-editing assumes that points from different classes that are close to apparent boundaries between them contain "noise" (i.e., points that "cross" the apparent boundaries between classes, making the labeled clusters seem mixed), and should therefore be discarded. This group of methods include Wilson's method ${ }^{18}$ and Multiedit. ${ }^{19,20}$ Both schemes are based on ruling out misclassified objects. In Wilson's method, the $k$-nn algorithm (Wilson recommends $k=3$ ) is run once on $X$. All misclassified objects are marked for deletion during the run, and are deleted from $X$ after the run to produce the prototype set $\mathbf{V}_{s}$.

Multiedit, a theoretically justified version of Wilson's method, is asymptotically Bayes optimal. That is, when the numbers of samples and iterations tends to infinity, the 1-np classifier on the resultant $\mathbf{V}_{s}$ is equivalent to the Bayes classifier. Multiedit, however, is not suitable for small data sets with overlapping clusters, whereas Wilson's method works well in these cases. Moreover, Wilson's method is appealingly simple.

Error-editing methods have no explicit connection to either the resubstitution or generalization error rate performance of the 1-np classifier based on the resultant $\mathbf{V}_{s}$. Asymptotic properties do not guarantee good performance when $\mathbf{V}_{s}$ has to be selected from a finite $X$. The cardinality of $\mathbf{V}_{s}$ is not explicitly taken into account. Many early techniques for editing 1-nn training data are summarized in Dasarathy. ${ }^{15}$ Our preliminary experiments indicated that the methods of Hart, Wilson, and Randomized Hart were not very effective in terms of either accuracy or data set reduction. In the finalized experiments reported in Section V we used Wilson's method followed by Hart's C-nn (called Wilson + Hart).

Hybrids of condensation and error-editing are also known. Editing techniques are often followed by condensing techniques. Editing "cleans up" the input data, yielding a $\mathbf{V}_{s \text {, initial }}$ that supposedly contains only "easy" points in it. Then a condensation method reduces $\mathbf{V}_{s \text {, initial }}$ to a possibly smaller number of relevant final prototypes, say $\mathbf{V}_{s \text {,final }}$. Ferri ${ }^{21}$ proposes a third step: Multiedit is used for phase 1 "clean up"; Hart's C-nn for phase 2 condensation; $\mathbf{V}_{s, \text { final }}$ is then used to reclassify all the original points in $X$, and the newly labeled data set, say $X^{\prime}$, is used with the DSM method to further refine the classification boundary.

Interestingly, condensation and error-editing rely on different ideas and implement different strategies (retain or discard boundary objects) aimed at the same general goal: finding the smallest possible $\mathbf{V}_{s}$ with the highest possible 1-np accuracy. There is a third group of methods for prototype selection that attempt to achieve the same goal through criterion-driven combinatorial optimization. We call this group of methods "boundary indifferent," because only the mathematical goal is specified, so an algorithm "decides" whether or not to retain boundary prototypes. An instant benefit from this third approach is that the cardinality of $\mathbf{V}_{s}$ and the classification accuracy can be balanced through the criterion used. The basic combinatorial optimization problem to be solved by this approach (and the one used by our genetic algorithm search method in the 
numerical experiments of Section V) is

$$
\underbrace{\max }_{\mathbf{V}_{s} \in P(X)}\left\{J\left(\mathbf{V}_{s}\right)\right\}=\underbrace{\max }_{\mathbf{V}_{s} \in P(X)}\left\{\left(1-E_{\mathrm{np}}\left(X ; \mathbf{V}_{s}\right)\right)-\alpha \frac{\left|\mathbf{V}_{s}\right|}{|\mathbf{X}|}\right\}
$$

where $P(X)$ is the power set of $X,\left(1-E_{\mathrm{np}}\left(X ; \mathbf{V}_{s}\right)\right)$ is the resubstitution classification accuracy achieved by the 1-np design based on $\mathbf{V}_{s}$, and $\alpha$ is a positive coefficient which essentially determines the tradeoff between accuracy and cardinality. The function $J$ in (4) is a typical fitness function for data editing; see Refs. 6 and 16 for other useful functional forms. Next, we briefly review three methods from this third group that all make use of (4) to evaluate potential solutions to the selection problem: random selection, GA-based search, and Tabu search.

Random selection (RS): The desired cardinality $n_{p}$ of $\mathbf{V}_{s}$ and the number of trials $T$ are specified in advance. Then $T$ random subsets of $X$ of cardinality $n_{p}$ are generated, and the one with the smallest error rate is retained as $\mathbf{V}_{s}$. Skalak calls this method a Monte Carlo simulation. ${ }^{22}$ In both Refs. 6 and 22 , random search is reported to work unexpectedly well.

Genetic algorithms (GAs): Editing the training data with GAs has been discussed by Chang and Lippmann, ${ }^{23}$ Kuncheva, ${ }^{24,25}$ and Kuncheva and Bezdek. ${ }^{6}$ Selection of a subset can be naturally encoded in terms of GAs (and more generally, the evolutionary search paradigm). A binary-valued chromosome $C$ represents a subset $S$ of the labeled data set $X$. The $i$ th bit in $C$ corresponds to $\mathbf{x}_{i}$ from $X$ and has value one if $\mathbf{x}_{i}$ is included in $S$, and 0 , otherwise. An example of this type of encoding is shown in Figure 4.

Our GA approach for the numerical experiments of the next section assesses a population of chromosomes in each generation. An initial population of $n_{\text {pop }}$ subsets of $X$ like $S$ in Figure 4 is randomly generated. For each $S$, each bit is 0 or 1 with probability $P_{\text {ini }}$, which regulates the cardinality of the initial population. Various members of the initial population can have different cardinalities. Each chromosome is evaluated by the fitness function $J$ in (4). The whole population set is used to choose $n_{\text {pop }} / 2$ parent couples and each couple produces two children by uniform crossover; i.e., each pair of corresponding bits of the parent chromosomes are swapped with probability $P_{c}=0.5$. Higher or lower values for $P_{c}$ will increase the resemblance between the children and their parents. The set of offspring is then mutated, i.e., each bit of each offspring chromosome is altered with a (small) predefined probability $P_{m}$ (this probability was fixed at $P_{m}=0.1$ ). New chromosomes are assessed by the fitness function $J$, pooled with the parent population, and the $n_{\text {pop }}$ chromosomes from the pool with the highest $J$ values survive as the next generation (elitist strategy). The

$$
X=\left\{\mathbf{x}_{1}, \ldots, \mathbf{x}_{8}\right\} \quad(+) \quad C=\begin{array}{|l|l|l|l|l|l|l|l|}
0 & 1 & 1 & 0 & 0 & 1 & 0 & 0
\end{array} \Rightarrow S=\left\{\mathbf{x}_{2}, \mathbf{x}_{3}, \mathbf{x}_{6}\right\}
$$

Figure 4. Binary encoding of a subset of $X$. 
algorithm terminates after $T$ generations, where $T$ is picked in advance ( $T=100$ in our experiments). The best chromosome from the last generation (the chromosome with the highest $J$ ) defines the prototype set $\mathbf{V}_{s}$, and the number of prototypes is thus determined "automatically," in the auto- $n_{p}$ sense as used here. You should realize, however, that there are other parameters the user does have to choose with a method such as this one, and $\left|\mathbf{V}_{s}\right|=n_{p}$ implicitly depends on your choices for these other parameters.

Our GA model with the selected parameter values is close to random selection. The reason for these parameter choices was threefold: (1) RS works reasonably well; (2) our GA model is simpler than "classical" ones which use more sophisticated selection schemes, e.g., the roulette wheel strategy; and (3) due to the enhanced random component, our GA is less likely to depend heavily on the initialization than classical schemes. But the price we pay for this is that our GA is also less likely to terminate quickly at local extrema of $J$. Our computational experience is that a few runs of this simpler scheme can lead to a reasonably good solution.

An even simpler evolutionary algorithm for data editing called "random mutation hill climbing" was proposed by Skalak. ${ }^{22}$ Instead of evolving a population of chromosomes simultaneously, only one chromosome evolves (should we call it survival of the only?), and only the mutation operation is performed on it. The best set in $T$ mutations is returned as $\mathbf{V}_{s}$. The evolutionary schemes given in Refs. 6 and 22 are both heuristic; it is debatable which is the better of the two. GA conducts a larger search by keeping different subsets of candidates in its early stages. On the other hand, the random mutation method is really simple, and, like the GA discussed in Ref. 6, is shown in Ref. 22 to outperform RS.

Tabu search (TS): Tabu search is an interesting alternative to the heavily randomized methods in the boundary-indifferent group. ${ }^{16}$ In this scheme the number of iterations $T$ is fixed but the cardinality $n_{p}$ is not. Similar to random mutation hill climbing, TS operates on one subset only, called the current solution $S$, which is represented as in Figure 4. A tabu vector of length $|X|$ is set up with all of its entries initially set to zero. An entry of 0 in the $k$ th place in the Tabu vector indicates that $\mathbf{x}_{k}$ can be added or deleted from $S$, while an entry that is greater than 0 prohibits a change in the status of $\mathbf{x}_{k}$. A parameter called tabu tenure $\left(T_{t}\right)$ is specified, defining the number of iterations before a change of any previously altered bit is allowed. An initial subset is picked as $S$, stored as the initial approximation of $\mathbf{V}_{s}$, and evaluated by $J\left(\mathbf{V}_{s}\right)$. The TS algorithm operates as follows.

First, all neighboring subsets to $S$ are evaluated by the criterion function $J$. A neighbor to $S$ in the subset selection problem is any subset of $X$ that differs from $S$ by just one element. For example, the eight neighbor solutions to $S$ in Figure 4 are obtained by altering one bit of the chromosome at a time: $\left\{\mathbf{x}_{1}, \mathbf{x}_{2}, \mathbf{x}_{3}, \mathbf{x}_{6}\right\},\left\{\mathbf{x}_{3}, \mathbf{x}_{6}\right\},\left\{\mathbf{x}_{2}, \mathbf{x}_{6}\right\},\left\{\mathbf{x}_{2}, \mathbf{x}_{2}, \mathbf{x}_{4}, \mathbf{x}_{6}\right\},\left\{\mathbf{x}_{2}, \mathbf{x}_{3}, \mathbf{x}_{5}, \mathbf{x}_{6}\right\},\left\{\mathbf{x}_{2}, \mathbf{x}_{3}\right\}$, $\left\{\mathbf{x}_{2}, \mathbf{x}_{3}, \mathbf{x}_{6}, \mathbf{x}_{7}\right\}$, and $\left\{\mathbf{x}_{2}, \mathbf{x}_{3}, \mathbf{x}_{6}, \mathbf{x}_{7}\right\}$. The neighbor subset $\hat{S}$ that yields the highest value of $J$ is called the winning neighbor. If $J\left(\mathbf{V}_{\hat{s}}\right)>J\left(\mathbf{V}_{s}\right), \hat{S}$ replaces $S$, regardless of the tabu vector, and $\mathbf{V}_{s}$ and $J\left(\mathbf{V}_{s}\right)$ are updated. If $J\left(\mathbf{V}_{\hat{s}}\right) \leq J\left(\mathbf{V}_{s}\right)$, the tabu vector is checked. If the move from $S$ to $\hat{S}$ is allowed (the tabu value 
$T_{t}=0$ ), the move is made anyway, and the corresponding slot of the tabu vector is set to $T_{t}$. Thus, tabu search does not necessarily have the ascent property. All other nonzero values in the tabu vector are then decreased by one. Different criteria can be applied for terminating the algorithm. Four methods for picking the initial solution are proposed in Ref. 16.

(1) Maximal. The initial set is all of $X$.

(2) Minimal. One prototype from each class is randomly picked from $X$.

(3) Condensed. Hart's C-nn is run on $X$, and the resultant consistent set $\mathbf{V}_{s}$ is used as the initial solution.

(4) Constructive. Starting from a minimal solution (second option), deletion of elements is banned until a consistent set is obtained. Then normal tabu search is resumed.

The authors' of Ref. 16 favor the constructive initialization. In the experiments of Section V we used their constructive initialization but did not wait until a consistent set was obtained. The initial incremental phase was terminated after a prespecified number of iterations (20 in all of our experiments).

\section{PROTOTYPE REPLACEMENT}

This section contains brief overviews of the seven methods used in our numerical experiments. Following the architecture of Figure 3, we have subsections for presupervised and postsupervised methods.

\section{A. Presupervised Designs}

Learning vector quantization (LVQ): The LVQ family comprises a large spectrum of competitive learning schemes. ${ }^{9}$ One of the basic designs that can be used for prototype generation is the LVQ1 algorithm. An initial set of labeled prototypes is picked by first specifying $n_{p} \geq c$. Then $n_{p}$ elements are randomly selected from $X$ to be the initial prototypes, so each class is represented by at least one prototype. LVQ1 has three additional parameters specified by the user: the learning rate $\alpha_{k} \in(0,1)$, a constant $\eta \in(0,1)$, and the terminal number of iterations $T$. The standard competitive learning update equation is then used to alter the prototype set. If the closed prototype for input $\mathbf{x}_{k}$ is the vector $\mathbf{v}_{i \text {, old }}$, the LVQ1 update strategy is

$$
\mathbf{v}_{i, \text { new }}=\mathbf{v}_{i, \text { old }}+\alpha_{k}\left(\mathbf{x}_{k}-\mathbf{v}_{i, \text { old }}\right) \quad \text { when } \ell\left(\mathbf{v}_{i, \text { old }}\right)=\ell\left(\mathbf{x}_{k}\right)
$$

or

$$
\mathbf{v}_{i, \text { new }}=\mathbf{v}_{i, \text { old }}-\alpha_{k}\left(\mathbf{x}_{k}-\mathbf{v}_{i, \text { old }}\right) \quad \text { when } \ell\left(\mathbf{v}_{i, \text { old }}\right) \neq \ell\left(\mathbf{x}_{k}\right)
$$

Equation (5a) rewards the winning prototype for getting the correct label by letting it migrate towards the input vector, while Eq. (5b) punishes the winning prototype for not labeling the current input correctly by repelling it away from the input vector. In our experiments, the learning rate was updated after each presentation of a new vector using the formula $\alpha_{k+1}=\eta \alpha_{k}, k=1, \ldots, n-1$; 
and was restored to the initial, user specified value of $\alpha_{1}$ at the end of each pass through $X$. Before each new pass through LVQ1, $X$ is randomly permuted to avoid dependence of the extracted prototypes on the order of inputs. LVQ1 terminates when either (i) there are no misclassifications in a whole pass through $X$ (and hence, the extracted prototypes are a consistent reference set); or (ii) the prespecified terminal number of iterations is reached. The set of prototypes at termination of LVQ1 is $\mathbf{V}_{r}$.

Decision surface mapping (DSM): Geva and Sitte's DSM ${ }^{13}$ is a variant of LVQ which they assert better approximates classification boundaries of the training data than LVQ does. These authors say the price for better classification rates is that the DSM is somewhat less stable than standard LVQs. The only difference between LVQ1 and DSM is that a punishment-reward step is taken only in case of misclassification of the current element of $X$. In LVQ1 the winning prototype is either punished or rewarded, depending on the outcome of the 1-np label match to the input. But in DSM, Eqs. (5) are both implemented only if misclassification occurs. Thus, when the 1-np rule produces the correct label, no update is made, but when misclassification occurs, both (5a) and (5b) are implemented. In this case the winner (from the wrong class) is punished by (5b) and the nearest prototype from the same class as the current input is identified and rewarded by (5a).

Both LVQ1 and DSM operate with a fixed number of prototypes chosen by the user, so are user- $n_{p}$ methods. An auto- $n_{p}$ modification of LVQ that can prune and relabel prototypes was proposed by Odorico, ${ }^{12}$ who called it LVQTC.

LVQ with training counters (LVQTC): Odorico's LVQTC algorithm operates similarly to LVQ1. The amount that the winning prototype $\mathbf{v}_{i, \text { old }}$ is updated depends on the distance to input $\mathbf{x}_{k}$ and the history of the prototype. A prototype's historical importance is determined by the number of times it has been the winner. The procedure is initialized as is LVQ1, with the added twist that an empty training counter with $c$ slots (one for each class) is attached to each prototype. When $\mathbf{x}_{k}$ is from class $i$, the $i$ th entry of the training counter of the nearest prototype will be incremented, regardless of the true label of that prototype. If the winning prototype is from class $i$ (shares the same label as the input vector), then a slight variation of the reward equation (5a) is applied to the winning prototype $\mathbf{v}_{i \text {, old }}$ :

$$
\mathbf{v}_{i, \text { new }}=\mathbf{v}_{i, \text { old }}+\frac{\alpha_{k}}{q_{i}}\left(\mathbf{x}_{k}-\mathbf{v}_{i, \text { old }}\right) \quad \text { when } \ell\left(\mathbf{v}_{i, \text { old }}\right)=\ell\left(\mathbf{x}_{k}\right)
$$

where $q_{i}$ is the total number of times that $\mathbf{v}_{i}$ has won the competition (i.e., the sum of all entries in its training counter vector). In (6) $\alpha_{k}$ is updated as in (5), but is not restored to its initial value at the end of each pass through $X$. The rationale for this treatment of the learning rate is that prototypes which have been modified many times have already found a good place in the feature space and should be less affected by subsequent inputs. This strategy can be thought of as a "decreasing reward"- not as strong as punishing the prototype for being wrong as in (5b) by sending it away-but more like making it less welcome the 
more times it shows up at your doorstep. Equation (6) is the same as the update equation for one of the earliest competitive learning models, viz., sequential hard c-means, ${ }^{26}$ for the particular choice $\alpha_{k} \equiv 1$. Odorico may or may not have recognized this, but in any case added some novel heuristics to the original algorithm which seem both justifiable and useful.

At the end of each pass through $X$ the prototype counters $\left\{q_{i}\right\}$ are compared to a user-specified retention threshold $Q$. If $q_{i}<Q$ (indicating that $\mathbf{v}_{i}$ has not won the competition "often enough" to merit retention), $\mathbf{v}_{i}$ is removed from $\mathbf{V}$. The prototypes which survive the retention test are checked to see if relabeling is needed. If the number of "calls" to a prototype from a wrong class exceeds $Q$, the prototype is relabeled to that class, and is placed at the centroid of all the vectors from that class which called it. Although this algorithm has many heuristics and depends critically on the choice of $Q$, it has the advantage that the final prototypes $\mathbf{V}_{r}$ provide soft class labels that are easily defined by the training counters. These soft labels can be used to guide classification decisions.

Bootstrap editing. Four simple and intuitive bootstrap editing methods are proposed in Hamamoto et al., ${ }^{27}$ all of which are presupervised, $R$-prototype extraction models. For the one that we use in Section V [which we will identify as BTS(3)], three parameters are user-specified: the number of nearest neighbors $k$, the desired number of prototypes $n_{p}$, and the number of random trials $T$. A random sample of size $n_{p}$ is then drawn from $X$. Each data point is replaced by the mean of its $k$-nearest neighbors from $X$ with the same class label. The 1-np classifier is run on $X$ using the new set of labeled prototypes. The best set from $T$ runs is returned as the final $\mathbf{V}_{r}$. In our experience, Hamamoto et al.'s method gives pretty nice results. This bootstrapping scheme is a simple, fast, and unashamedly random way to get presupervised $R$-prototypes that seem to provide a low resubstitution error rate.

\section{B. Postsupervised Designs}

Methods in this category disregard the class labels during training, and use $X$ as if it were unlabeled to find a set $\mathbf{V}_{r}$ of algorithmically labeled prototypes. The prototypes are then relabeled using the training data labels. Methods in this group can be implemented in the presupervised mode by using the data from one class at a time, since this obviates the necessity for the relabeling step. When working with the data class by class, the prototypes that are found for each labeled class already have the assigned physical labels. The $c$ subsets of prototypes are then pooled to form $\mathbf{V}_{r}$. We focus here on postsupervised designs. Your intuition probably tells you that presupervised prototype extraction $\left(\mathbf{V}_{s}\right.$ as opposed to $\mathbf{V}_{r}$ ) will usually produce better 1-np classifiers than postsupervised models. We will keep an eye on this supposition as we conduct numerical experiments in the next section (cf. Kuncheva and Bezdek, ${ }^{6}$ Kohonen, ${ }^{8}$ and Diamantini and Spalvieri ${ }^{10}$ ).

Vector Quantization (VQ): Vector quantization has been used to find prototypes for many years. For example, Xie et al. ${ }^{28}$ used it (in the presupervised model) to support a version of $k$-nearest neighbor and Parzen classifier 
designs. In this study we adhere to the basic algorithm and apply it to the whole data set in the postsupervised mode. VQ starts with the user specifying $n_{p}$, and randomly selecting an initial set of $n_{p}$ unlabeled prototypes from $X$. The closest prototype is always rewarded according to the update equation

$$
\mathbf{v}_{i, \text { new }}=\mathbf{v}_{i, \text { old }}+\alpha_{t}\left(\mathbf{x}_{k}-\mathbf{v}_{i, \text { old }}\right)
$$

The learning rate in (7) is indexed on $t$ instead of $k, t$ being the iteration counter (one iteration is one pass through $X$ ). The update rule we used is $\alpha_{t+1}=(1-(t / T)) \alpha_{t}, t=1, \ldots, T$, where $T$ is a prespecified maximum number of passes through $X$. Termination is achieved by reaching $T$ or by satisfying a measure of closeness of successive iterates to a user-defined termination threshold, i.e., $\left\|\mathbf{V}_{k+1}-\mathbf{V}_{k}\right\| \leq \varepsilon$. To assign physical labels to the prototypes, the 1-np rule is applied to $X$ using the extracted prototypes. The number of winners for each prototype from all $c$ classes are counted. Finally, the most represented class label is assigned to each prototype. Let $n_{i j}$ be the number of elements from class $j$ associated with prototype $\mathbf{v}_{i}$ via the 1-np rule. Class label $k$ is assigned to prototype $\mathbf{v}_{i}$ when it holds the majority of votes:

$$
n_{i k}=\underbrace{\max }_{1 \leq j \leq c}\left\{n_{i j}\right\} \Rightarrow \ell\left(\mathbf{v}_{i}\right)=\mathbf{e}_{k}
$$

Ties in (8) are broken randomly. It is not difficult to show that this relabeling strategy guarantees the smallest number of misclassifications of the resultant 1-np classifier on $X$. This relabeling scheme is used in all of our other postsupervised designs.

Generalized Learning Vector Quantization-Fuzzy GLVQ-F: GLVQ-F is an unsupervised method for finding prototypes which is similar to VQ but at each step all prototypes are updated. The update formula for the special case of weighting exponent $m=2$ as given in Ref. 29 is

$$
\mathbf{v}_{i, \text { new }}=\mathbf{v}_{i, \text { old }}+u_{i} \alpha_{t}\left(\mathbf{x}_{k}-\mathbf{v}_{i, \text { old }}\right)
$$

where

$$
u_{i}=\sum_{j=1}^{c}\left(\frac{\left\|\mathbf{x}-\mathbf{v}_{i}\right\|^{2}}{\left\|\mathbf{x}-\mathbf{v}_{j}\right\|^{2}}\right) \quad 1 \leq i \leq c
$$

The rest of the GLVQ-F algorithm is the same as our specification of VQ. See Ref. 29 for limit analysis of GLVQ-F, which reduces to VQ under certain conditions.

Clustering and relabeling: Instead of finding prototypes by sequential competitive learning, we can cluster $X$ with any batch clustering algorithm that generates cluster centers, and take the centroids as $\mathbf{V}_{r}$. This approach can be useful if the centroids are "good" representatives of the clusters in the data (of course, we will not be able to ascertain this for data that have more than $p=3$ features). Good candidates include the $c$-means methods. ${ }^{1,30} X$ is clustered 
Table I. Eleven (of zillions!) methods for finding prototypes.

\begin{tabular}{|c|c|c|c|c|}
\hline$\#$ & Acronym & $\mathrm{S}$ or $\mathrm{R}$ & Pre/Post & $n_{p}$ \\
\hline $\mathrm{c} 1$ & $\mathrm{~W}+\mathrm{H}$ & (S) & $\mathrm{P}$ & (A) \\
\hline $\mathrm{c} 2$ & GA & (S) & $\mathrm{P}$ & (A) \\
\hline c3 & RND & (S) & $\mathrm{P}$ & (U) \\
\hline c4 & Tabu & (S) & $\mathrm{P}$ & (A) \\
\hline c5 & LVQI & (B) & $\mathrm{P}$ & (U) \\
\hline c6 & DSM & (R) & $\mathrm{P}$ & (1) \\
\hline c7 & LVQTC & (B) & $\mathrm{P}$ & (A) $\downarrow$ \\
\hline c8 & BTS(3) & (R) & $\mathrm{P}$ & (1) \\
\hline c9 & VQ & (R) & (p) & (1) \\
\hline $\mathrm{c} 10$ & GLVQ-F & (B) & (p) & (U) \\
\hline c11 & $\mathrm{HCM}$ & (B) & (p) & (A) $\downarrow$ \\
\hline
\end{tabular}

disregarding the class labels. The centroids are taken as $\mathbf{V}_{r}$. The relabeling step assigns a class label to each cluster according to (8). Our experiments use only classical hard $c$-means. ${ }^{1}$

Summarizing, the 11 classifier methods we chose for prototype extraction are listed in Table I. The notation in the last three columns in Table I is used in Figures 9 through 12: selection $=(\mathrm{S})$, replacement $=(\mathbb{R})$, presupervised $=\mathrm{P}$, postsupervised $=(\mathbf{p})$, auto- $n_{p}=(\mathrm{A})$ and user- $n_{p}=$ (U). The notation (A) $\downarrow$ in Table I means that the algorithm can only decrease the initially specified number of prototypes. LVQTC prunes prototypes after each pass through $X$, and the HCM implementation (Duin ${ }^{31}$ ) can return a number of clusters that is smaller than the number of classes in the labeled data.

Why these 11 methods? Our choice was guided by two criteria: (i) the methods should be simple and effective, with a relatively small number of parameters to specify; and (ii), as much of the methodological spectrum shown in Figure 3 as possible should be represented.

\section{THE DATA SETS AND NUMERICAL EXPERIMENTS}

We use two small, artificially generated data sets, and two data sets from real application domains. Characteristics of the four data sets are shown in Table II.

(A) Cone-torus data. This is a $c=3$ class dataset with $n=400$ training points $\left(X_{\text {tr }}\right)$ in $\Re^{2}$ generated from three differently shaped distributions: a cone (92 training data), half a torus (99 training data), and a normal distribution (209 
Table II. Characteristics of the four data sets used in our computational experiments.

\begin{tabular}{lccccc}
\hline Name & $\begin{array}{c}p \\
\text { \# of } \\
\text { Features }\end{array}$ & $\begin{array}{c}c \\
\text { \# of } \\
\text { Classes }\end{array}$ & $\left|X_{\text {tr }}\right|$ & $\left|X_{\text {test }}\right|$ & Electronic Access \\
\hline Cone-Torus & 2 & 3 & 400 & 400 & $\begin{array}{c}\text { http://www.bangor.ac.uk/ } \\
\sim \text { mas00a/Z.txt }\end{array}$ \\
$\begin{array}{l}\text { Normal } \\
\text { mixture }\end{array}$ & 2 & 2 & 250 & 1000 & $\begin{array}{c}\text { http://www.stats.ox.ac.uk/ } \\
\text { ripley/PRNN/ } \\
\text { Phoneme }\end{array}$ \\
Satimage & 5 & 2 & 500 & 4904 & $\begin{array}{c}\text { ftp.dice.ucl.ac.be, directorypub/ } \\
\text { neural-nets/ELENA/ } \\
\text { ftp.dice.ucl.ac.be, directorypub/ } \\
\text { neural-nets/ELENA/ }\end{array}$ \\
\hline
\end{tabular}

training data) with prior probabilities for the corresponding classes of $0.25,0.25$, and 0.5 . A separate test data set ( $X_{\text {test }}$ ) with 400 more points generated from the same distributions is also provided.

(B) Normal mixtures data. This data set is used by Ripley ${ }^{32}$ to illustrate various classification techniques. The training data consist of 125 two-dimensional samples drawn from a mixture of two 2-variate normal distributions with the same covariance matrix. A labeled test set containing 1000 more points drawn from the same mixture distribution is also provided. Ripley asserts that the class distributions have been chosen so that the best possible error rate on the test data is about $8 \%$.

(C) Phoneme data. The Phoneme data set consists of 5404 five-dimensional vectors characterizing two classes of phonemes: nasals $(70.65 \%)$ and orals $(29.35 \%)$. A series of classification results based on the Phoneme data are presented in Holmstrom et al. ${ }^{33}$ who report classification accuracy that varies between 11-25\%, with the first half of the data set used for training and the second half for testing. In our experiments we used the first 500 data points for training and the remaining 4904 for testing.

(D) Satimage data. This data set, generated from the Landsat Multi-Spectral Scanner image data, consists of 6435 pixels, each of which has 36 intensities attached to it. The 36 features comprise 4 spectral bands, and each center pixel provides 9 intensities from its $3 \times 3$ neighborhood. Pixels are crisply labeled into one of $c=6$ classes, and are presented in random order in the database. The classes are: red soil $(23.82 \%)$, cotton crop $(10.92 \%)$, grey soil $(21.10 \%)$, damp grey soil $(9.73 \%)$, soil with vegetation stubble $(10.99 \%)$, and very damp grey soil $(23.43 \%)$. Our experiments are based on using the 4 features 17, 18, 19, and 20, which are the features recommended by the designers of the database. The first 500 points in this feature subset of the Satimage data were used for training, and the remaining 5935 points in these four features were used for testing.

Our experiments were not configured to find the best classification accuracy that might be possible for a given data set. Rather, our objective here is to use the four data sets as a means for comparing various aspects of the 11 prototype extraction methods when the prototypes are used as the basis of the 1-np 
classifier. Various combinations of initialization parameters were tried in preliminary experiments and the most successful ones were picked for the reported comparisons. You can undoubtedly find better combinations of parameters for the various methods if time is not an obstacle. HCM was implemented using the version available in the PRTOOLS toolbox of Duin. ${ }^{31}$ The number of clusters returned by Duin's HCM can be smaller than the initial number $n_{p \text {, ini }}$ because some of the clusters may turn out to be degenerate (i.e., empty). Table III shows the experimental protocols that we used for each of the 11 methods.

The training and testing errors and the terminal number of prototypes $|\mathbf{V}|=n_{p}$ for the eleven 1-np classifier methods and four data sets are shown in Table IV. For GA, Tabu search, LVQ1, DSM, LVQTC, VQ, GLVQ-F, and $\mathrm{HCM}$, the best of five independent runs is reported. Wherever the best training error over different runs was tied, the average of the test errors over the tied runs is given. The best results among the 11 competing schemes for training error, testing error, and number of prototypes are highlighted by bold font in Table IV.

The last column in Table IV shows the \% reduction in $\left|X_{\text {tr }}\right|$ on replacement of $X_{\mathrm{tr}}$ by $\mathbf{V}_{s}$ or $\mathbf{V}_{r}$. For example, classifier $c 1$ in Table IV selects 36 of the 400 training points in the cone-torus data as prototypes, so the percent reduction = $100 *(400-36) / 400=91 \%$. Observe that $|\mathbf{V}|>c$ for all 11 methods. That is, all of these classifiers are multiple prototype classifiers, in the sense that there is at least one class in the training data which is represented by more than one prototype. This is consistent with both our intuition and earlier studies (cf. Ref. 30).

Figures 5 through 8 plot the test error rate (column 5 in Table IV) $E_{\text {np }} \doteq E_{\text {np }}\left(X_{\text {test }} ; \mathbf{V}\right)$ versus the number of terminal prototypes $|\mathbf{V}|=n_{p}$ (prespecified or found, column 6 in Table IV) for the 11 methods and four data sets. Each method produces a point in the two-dimensional space whose coordinate axes are $\left(n_{p}, E_{\mathrm{np}}\right)$. The closer a point is to the origin, the better the 1-np

Table III. Experimental protocols.

\begin{tabular}{|c|c|c|c|c|c|c|}
\hline \multirow{5}{*}{$\begin{array}{l}\# \\
\mathrm{c} 1 \\
\mathrm{c} 2 \\
\mathrm{c} 3 \\
\mathrm{c} 4\end{array}$} & \multirow{4}{*}{$\begin{array}{l}\text { Method } \\
\text { W + H } \\
\text { GA } \\
\text { RS }\end{array}$} & \multicolumn{5}{|c|}{ Initialization Parameters } \\
\hline & & $k=3$ & & & & \\
\hline & & $p s=10$ & $T=100$ & $P_{m}=0.1$ & $P_{\text {ini }}=0.1$ & $\alpha=0.5$ \\
\hline & & $T=500$ & $n_{p}=\{10,20\}$ & & & \\
\hline & Tabu & & $T=100(+20$ & preliminary & iterations) & \\
\hline & & $T_{t}(\mathrm{tabu} t$ & nure $)=3 \%$ of & he chromo & some length, & $\alpha=0.05$ \\
\hline $\mathrm{c5}$ & LVQ1 & $\alpha_{\text {ini }}=0.3$ & $\eta=0.8$ & $T=100$ & $n_{p}=20$ & \\
\hline c6 & DSM & $\alpha_{\text {ini }}=0.3$ & $\eta=0.8$ & $T=100$ & $n_{p}=20$ & \\
\hline c7 & LVQTC & $\alpha_{\mathrm{ini}}=0.3$ & $\eta=0.8$ & $T=100$ & $Q=20$ & $n_{p, \text { ini }}=20$ \\
\hline c8 & BTS(3) & $k=3$ & $T=100$ & & & \\
\hline c9 & VQ & $\alpha_{\mathrm{ini}}=0.3$ & $T=100$ & $n_{p}=20$ & & \\
\hline c10 & GLVQ-F & $\alpha_{\text {ini }}=0.3$ & $T=100$ & $n_{p}=20$ & & \\
\hline c11 & HCM & $n_{p, \text { ini }}=20$ & & & & \\
\hline
\end{tabular}


Table IV. Experimental results (best case) for the four data sets.

\begin{tabular}{clcccc}
\hline$\#$ & Method & $\begin{array}{c}\text { Training } \\
\text { Error [\%] }\end{array}$ & $\begin{array}{c}\text { Testing } \\
\text { Error [\%] }\end{array}$ & $n_{p}$ & $\begin{array}{c}\% \\
\text { Reduction }\end{array}$ \\
\hline $\begin{array}{c}\text { Cone-Torus } \\
\text { c1 }\end{array}$ & Wata: (cols. 4 and 5 plotted in Fig. 5) & & & \\
c2 & GA & 10.75 & 13.00 & 36 & 91 \\
c3 & RND & 10.00 & 14.50 & 77 & 80.75 \\
c4 & Tabu & 15.75 & 14.50 & 20 & 95 \\
c5 & LVQ1 & $\mathbf{9 . 5}$ & 14.75 & 21 & 94.75 \\
c6 & DSM & 22.25 & 14.50 & 20 & 95 \\
c7 & LVQTC & 17.00 & 20.75 & 20 & 95 \\
c8 & BTS(3) & 16.00 & 16.00 & $\mathbf{1 3}$ & 96.75 \\
c9 & VQ & 14.75 & 12.25 & 20 & 95 \\
c10 & GLVQ-F & 17.00 & 15.75 & 20 & 95 \\
c11 & HCM & 14.25 & 18.25 & 20 & 95 \\
\end{tabular}

Normal Mixture Data: (cols. 4 and 5 plotted in Fig. 6)

$\begin{array}{llrlll}\text { c1 } & \text { W + H } & 10.4 & 14.9 & 23 & 90.8 \\ \text { c2 } & \text { GA } & 8.8 & 11.3 & 42 & 83.2 \\ \text { c3 } & \text { RND } & 10.8 & 10.65 & \mathbf{1 0} & 96 \\ \text { c4 } & \text { Tabu } & \mathbf{8 . 4} & 12.9 & \mathbf{1 0} & 96 \\ \text { c5 } & \text { LVQ1 } & 10.0 & \mathbf{8 . 6} & 20 & 94 \\ \text { c6 } & \text { DSM } & 12.4 & 9.0 & 20 & 92 \\ \text { c7 } & \text { LVQTC } & 10.0 & 10.5 & 15 & 94 \\ \text { c8 } & \text { BTS(3) } & 10.4 & 10.2 & 20 & 92 \\ \text { c9 } & \text { VQ } & 12.0 & 11.80 & 20 & 92 \\ \text { c10 } & \text { GLVQ-F } & 12.0 & 9.2 & 20 & 92 \\ \text { c11 } & \text { HCM } & 11.2 & 9.5 & 19 & 92.4\end{array}$

Phoeneme Data: (cols. 4 and 5 plotted in Fig. 7)

$\begin{array}{llllll}\text { c1 } & \text { W + H } & 10.80 & 20.00 & 47 & 90.6 \\ \text { c2 } & \text { GA } & 11.80 & 20.84 & 98 & 80.4 \\ \text { c3 } & \text { RND } & 18.40 & 22.14 & 20 & 96 \\ \text { c4 } & \text { Tabu } & \mathbf{9 . 8 0} & \mathbf{1 8 . 8 0} & 20 & 96 \\ \text { c5 } & \text { LVQ1 } & 15.4 & 21.53 & 20 & 96 \\ \text { c6 } & \text { DSM } & 23.2 & 27.55 & 20 & 96 \\ \text { c7 } & \text { LVQTC } & 17.6 & 21.02 & \mathbf{1 0} & 98 \\ \text { c8 } & \text { BTS(3) } & 17.00 & 22.29 & 20 & 96 \\ \text { c9 } & \text { VQ } & 19.6 & 24.04 & 20 & 96 \\ \text { c10 } & \text { GLVQ-F } & 19.40 & 23.45 & 20 & 96 \\ \text { c11 } & \text { HCM } & 18.2 & 20.84 & 19 & 96.2\end{array}$

Satimage Data: cols. 4 and 5 plotted in Fig. 8)

$\begin{array}{llllll}\text { c1 } & \text { W }+ \text { H } & 11.4 & 16.16 & 32 & 93.6 \\ \text { c2 } & \text { GA } & 11.0 & 17.83 & 115 & 77 \\ \text { c3 } & \text { RND } & 17.4 & 19.51 & 20 & 96 \\ \text { c4 } & \text { Tabu } & \mathbf{1 0 . 4} & 16.41 & 24 & 95.2 \\ \text { c5 } & \text { LVQ1 } & 15.2 & 16.68 & 20 & 96 \\ \text { c6 } & \text { DSM } & 19.6 & 20.35 & 20 & 96 \\ \text { c7 } & \text { LVQTC } & 16.0 & 17.51 & \mathbf{1 6} & 96.8 \\ \text { c8 } & \text { BTS(3) } & 16.6 & 17.17 & 20 & 96 \\ \text { c9 } & \text { VQ } & 15.2 & \mathbf{1 5 . 8 6} & 20 & 96 \\ \text { c10 } & \text { GLVQ-F } & 15.6 & 16.34 & 20 & 96 \\ \text { c11 } & \text { HCM } & 14.4 & 17.07 & \mathbf{1 6} & 96.8\end{array}$




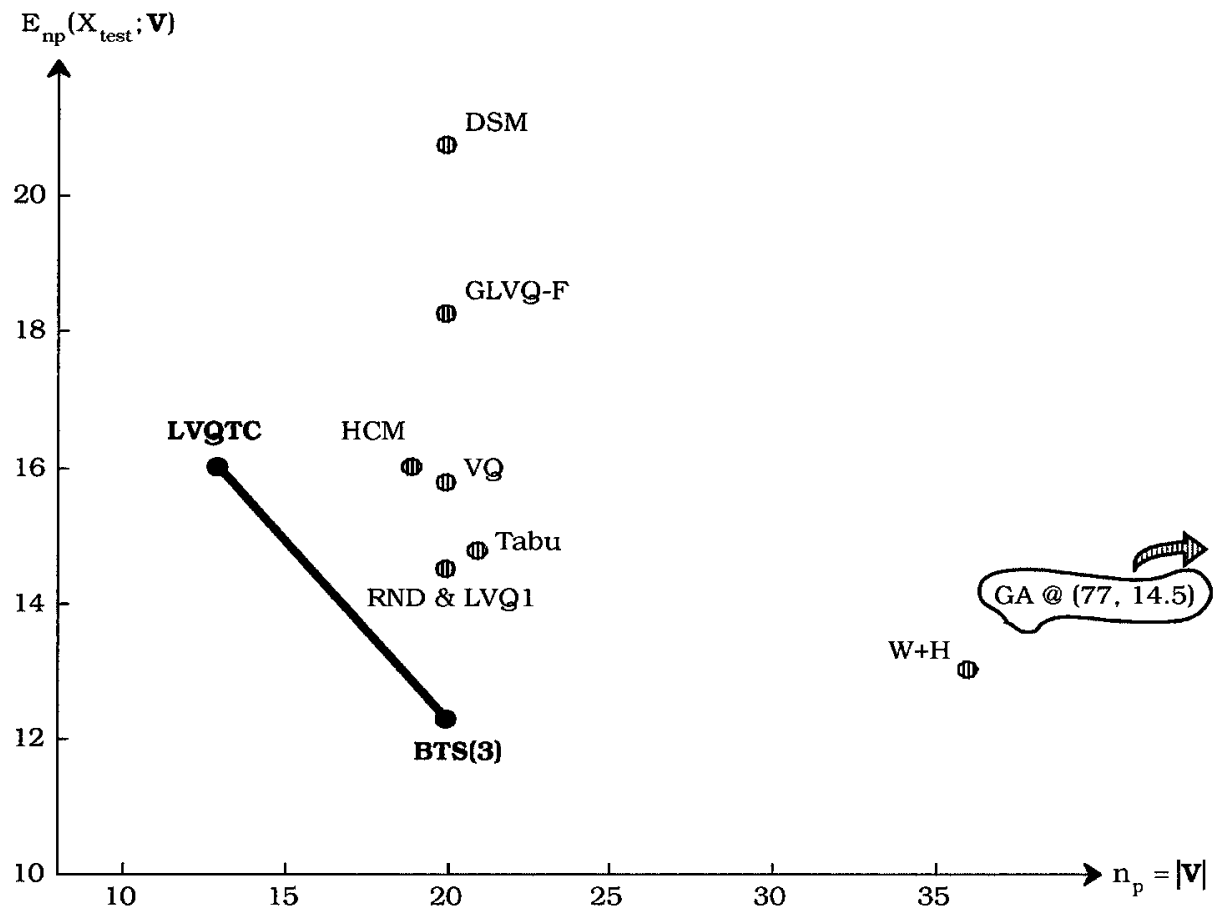

Figure 5. $n_{p}=|\mathbf{V}|$ vs. $E_{\mathrm{np}}\left(X_{\text {test }} ; \mathbf{V}\right)$ for the cone-torus data.

classifier, because such a classifier will have a small number of prototypes and also a small test error rate.

Figure 5 has coordinates for 10 of the 11 methods. The GA approach resulted in 77 prototypes for the cone-torus data, so we decided not to plot this point, to keep the scale in Figure 5 to a size where the other 10 methods can be seen more clearly. The same thing occurs with the other three data sets; the number of prototypes chosen by GA is much larger than those found by the other 10 methods, so we again left the GA coordinates off-scale. This also occurs for the $(\mathrm{W}+\mathrm{H})$ classifier in Figures 7 and 8 .

Figure 6 plots the same 10 methods as Figure 5 for training and testing with the normal mixture. The best classifiers in Figure 6 are RND and DSM, neither of which "wins" the best classifier contest depicted by Figure 5. This illustrates emphatically just how data dependent classifier design can be, and how ludicrous it is to look for a "best all-around" classifier design that generalizes well across a variety of different data regimes.

For the Phoneme data, the LVQTC and Tabu based 1-np designs produce the best results. Comparing Figure 7 to Figures 5 and 6 shows that over the first three data sets used for training and testing, our first repeat winner is the LVQTC based 1-np design. LVQTC occupies a place of distinction in both cases because it uses the minimum number of prototypes, but notice that this is 


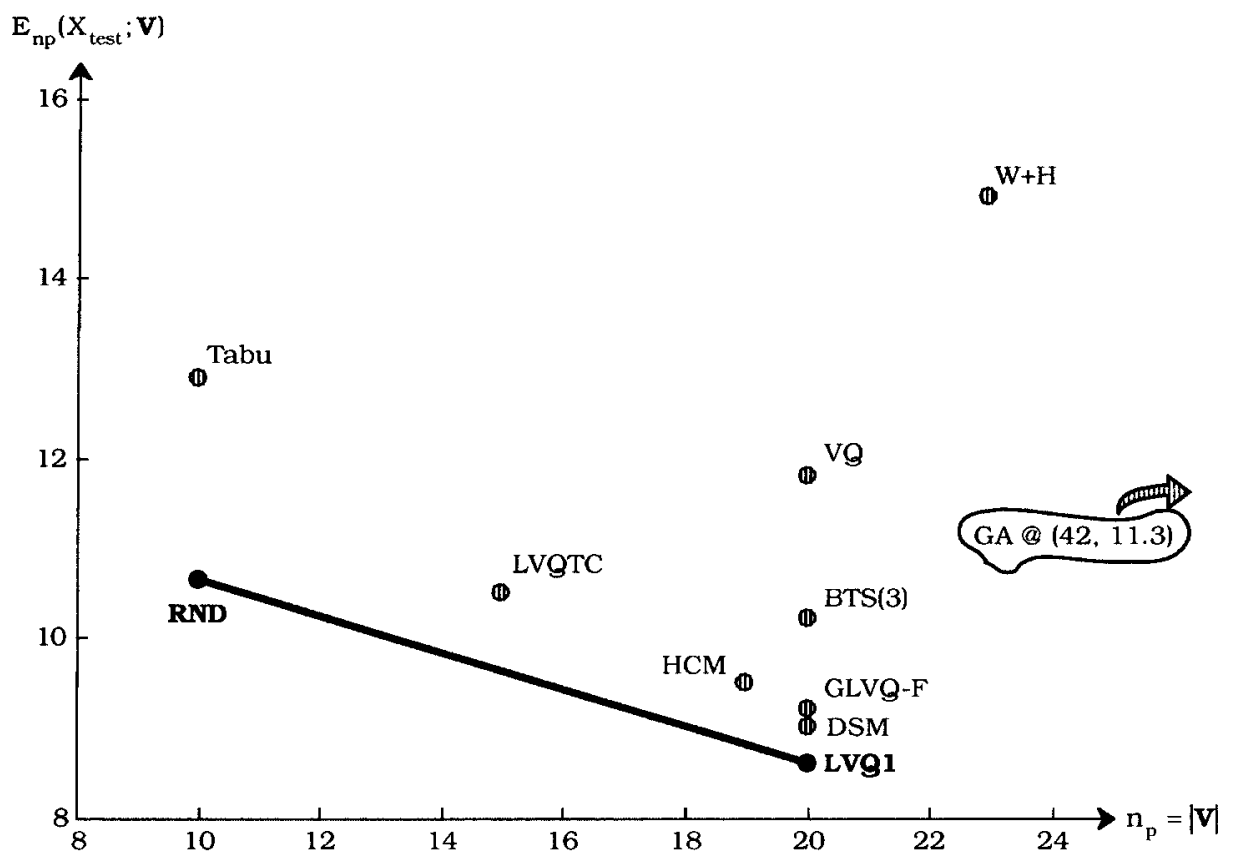

Figure 6. $n_{p}=|\mathbf{V}|$ vs. $E_{\mathrm{np}}\left(X_{\text {test }} ; \mathbf{V}\right)$ for the normal mixtures data.

achieved, for example, by sacrificing its testing error, which is worse for LVQTC in Figure 5 than for all but two of the competing 1-np designs.

Yet another pair of classifiers emerge as winners for the Satimage data, represented in Figure 8, where HCM and VQ based 1-np designs perform better in one coordinate or the other than the other nine classifier designs.

In some sense the best 1-np classifier overall will be the one closest to the origin in Figures 5 through 8 , since this design will have the minimum $\left[|\mathbf{V}|^{2}+\right.$ $\left.E_{\mathrm{np}}^{2}\right]$; the most efficient representation of the training data is perhaps the design that minimums $n_{p}=|\mathbf{V}|$; and the best design in terms of generalization error rate minimizes $E_{\mathrm{np}}$ alone. The tradeoff between minimal representation and maximal classification accuracy is evident from the fact that none of the classifiers studied have the smallest coordinates in both dimensions in Figures 5 through 8 . Winning designs for each of the four data sets are connected by lines in Figures 5 through 8 and summarized in Table $\mathrm{V}$.

The two ties in Table IV, $n_{p}=10$ with RND and Tabu, and $n_{p}=16$ with LVQTC and HCM, were resolved by choosing the classifier for Tables V and VI that was closer to the origin in Figures 6 and 8, respectively. While this gives us a way to declare a "winner" for these two data sets, please notice that there is hardly any difference between these two designs and the two that do not appear in Table $\mathrm{V}$ because of our tie-breaking strategy. 


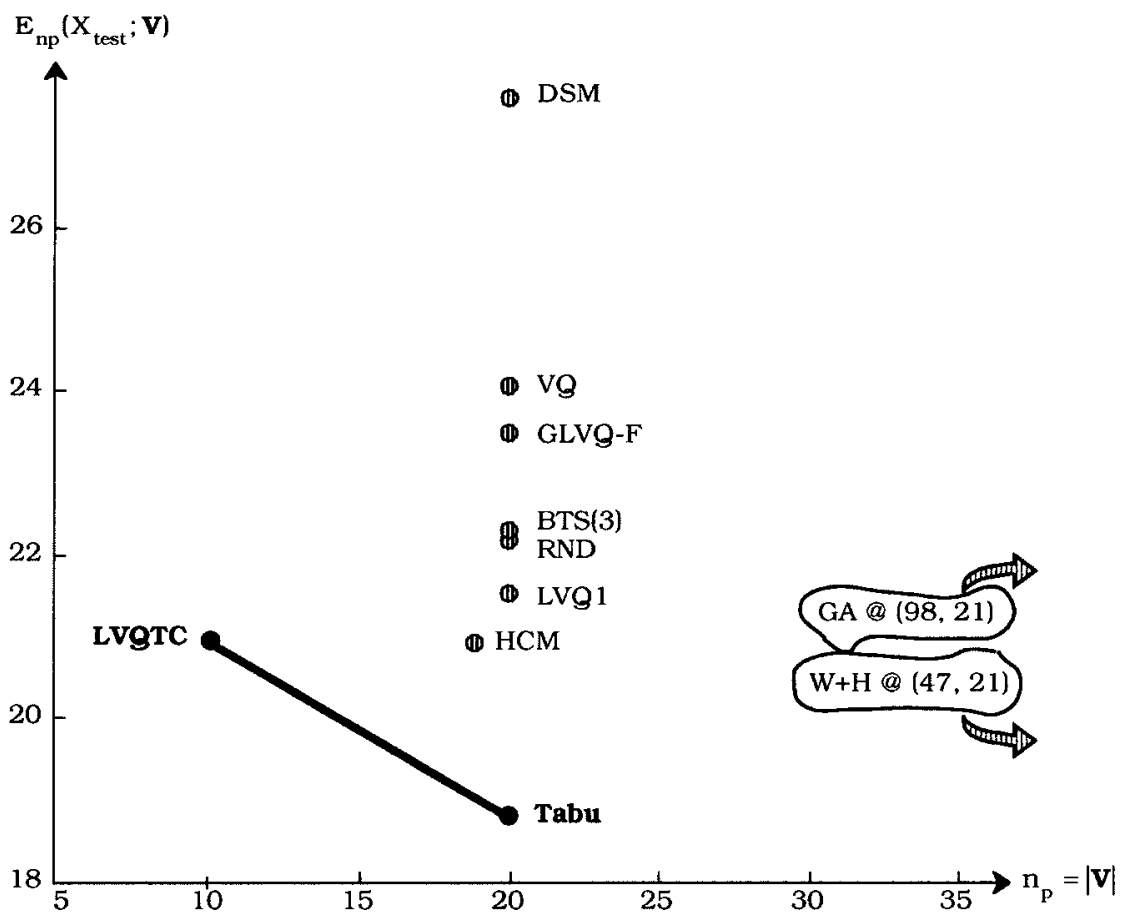

Figure 7. $n_{p}=|\mathbf{V}|$ vs. $E_{\mathrm{np}}\left(X_{\text {test }} ; \mathbf{V}\right)$ for the Phoneme data.

Now we return to the three characteristics of 1-np designs listed in Section I: (C1) Selection (S) $\left(\mathbf{V}_{s} \subseteq X\right)$ versus replacement $(\mathbb{R})\left(\mathbf{V}_{r} \not \subset X\right)$; (C2) Presupervised $\mathrm{P}$ versus postsupervised ( $\mathbf{p}$ ) designs; and (C3) User-defined $n_{p}$ (U) (user- $\left.n_{p}\right)$ versus algorithmically defined $n_{p}$ (A) $\left(\right.$ auto- $\left.n_{p}\right)$. One way to evaluate the overall effect of (C1), (C2), and (C3) on the classifiers is to combine the information in Tables I and V. Towards this end, column 1 of Table VI shows the winning 1-np classifier designs from Table V, while each row in Table VI has a set of three check $(\sqrt{ })$ marks corresponding to the three characteristics of the method from Table I.

The last row in Table VI shows the number of checks in each column, and the paired "scores" for each of $(\mathrm{C} 1)-(\mathrm{C} 3)$ give a very crude indication of relative efficacy of each coordinate in the pair. Since there are eight optimal points in Figures 5 through 8, each pair of coordinates sums to 8: e.g. $(S, R)=(2,6)$ in the last row of Table VI, so replacement methods achieved results at least as good or better than selection algorithms in six of the eight of the trials represented for the four data sets. As you think about this statement, bear in mind that the coordinate pairs on which it is based are in $\left(n_{p}, E_{\mathrm{np}}\right)$ space, and only one pair of coordinates per method appears in each of Figures 5 through 8. It is incorrect to infer from columns 3 and 4 of Table VI, for example, that $R$ 


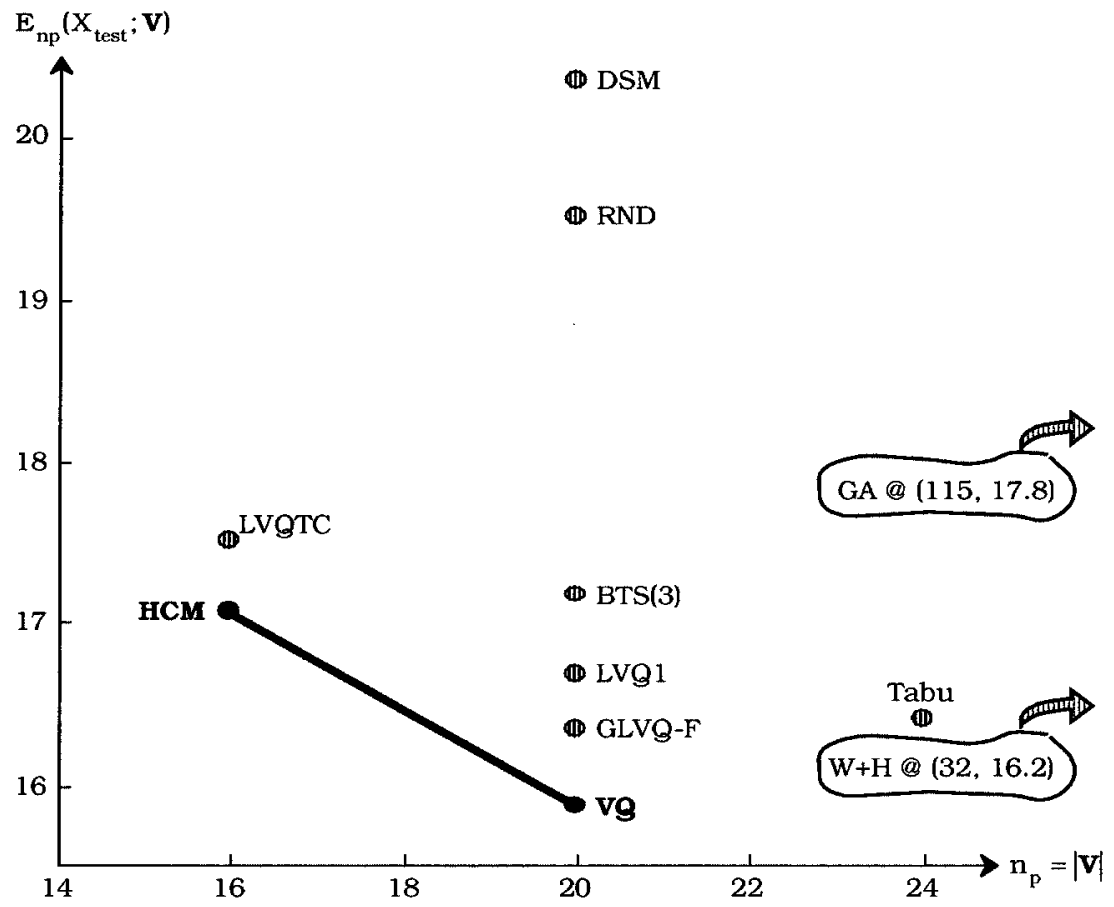

Figure 8. $n_{p}=|\mathbf{V}|$ vs. $E_{\mathrm{np}}\left(X_{\text {test }} ; \mathbf{V}\right)$ for the Satimage data.

methods achieve lower test error rates than $S$ methods in $75 \%$ of the trials. For instance, HCM posts a "win" in Figure 8, but there are five other classifiers that yield a lower testing error than HCM on the Satimage data. HCM appears in Table VI because none of its competitors can do better with as few prototypes. A better interpretation of the information in Tables V and VI bears in mind that Figures 5 through 8 all have an underlying (implicit, but very real) tradeoff going on between $n_{p}$ and $E_{\mathrm{np}}$.

Figures 9 through 11 try to address the relevance of (C1)-(C3) to 1-np classifier design in a slightly different way. In these three figures the horizontal axis is the ratio of $n_{p}$, the number of prototypes found or used, to the cardinality of the training set $X_{\text {tr }}$, averaged over the four data sets A, B, C, D, say $\bar{n}_{p}=$

Table V. Winning classifiers for the four data sets.

\begin{tabular}{lcrrrccc}
\hline Data Set & $\left|X_{\text {tr }}\right|$ & $\left|X_{\text {test }}\right|$ & $p$ & $c$ & \multicolumn{1}{c}{$\begin{array}{c}\min \\
n_{p}=|\mathbf{V}|\end{array}$} & $\begin{array}{c}\min \\
E_{\mathrm{np}}\end{array}$ & $|\mathbf{V}|^{2}+E_{\mathrm{np}}^{2}$ \\
\hline Cone-torus & 400 & 400 & 2 & 3 & $13:$ LVQTC & BTS(3) & LVQTC \\
Normal mixture & 250 & 1000 & 2 & 2 & $10:$ RND & LVQ1 & RND \\
Phoneme & 500 & 4904 & 5 & 2 & $10:$ LVQTC & Tabu & LVQTC \\
Satimage & 500 & 5935 & 4 & 6 & $16:$ HCM & VQ & HCM \\
\hline
\end{tabular}


Table VI. A summary of the winning designs for the four data sets.

\begin{tabular}{|c|c|c|c|c|c|c|c|}
\hline Method & Wins In & (S) & (B) & \begin{tabular}{|l|} 
\\
\end{tabular} & (p) & (U) & (A) \\
\hline LVQTC & Fig. 5 & & $\checkmark$ & V & & & V \\
\hline BTS(3) & Fig. 5 & & V & v & & ل & \\
\hline LVQ1 & Fig. 6 & & v & v & & v & \\
\hline RND & Fig. 6 & ل & & y & & v & \\
\hline Tabu & Fig. 7 & v & & v & & & l \\
\hline LVQTC & Fig. 7 & & $\sqrt{ }$ & V & & & V \\
\hline $\mathrm{HCM}$ & Fig. 8 & & v & & l & & v \\
\hline VQ & Fig. 8 & & V & & V & $\sqrt{ }$ & \\
\hline$\Sigma(\sqrt{ })$ & & 2 & 6 & 6 & 2 & 4 & 4 \\
\hline
\end{tabular}

$\sum_{i=1}^{4}\left(\left|\mathbf{V}_{i}\right| / 4 \cdot\left|X_{\mathrm{tr}, i}\right|\right)$, where index $i$ runs over the four data sets in question. The vertical axis in Figures 9 through 11 is the training error rate averaged over the four data sets, i.e., $\bar{E}_{\mathrm{np}}=\sum_{i=1}^{4} E_{\mathrm{np}}\left(X_{\mathrm{tr}, i} ; \mathbf{V}_{i}\right) / 4$. The GA based 1-np classifier does not appear in these three figures because its horizontal coordinate is much larger than any of the other classifiers, and we wanted to again show the comparisons at a scale which enables you to see differences among the classifiers close to the origin. The ten points in Figures 9 through 11 have the same coordinates in all three figures - what changes from Figure 9 to Figure 10 to Figure 11 is the label attached to each of the points. In Figure 9, we compare selection to replacement; in Figure 10 presupervised designs are compared to postsupervised designs; and in Figure 11, you can see how user- $n_{p}$ methods stack up to auto- $n_{p}$ methods. Classifiers which are closer to the origin in Figures 9 through 11 are again better than ones which are farther away from it.

Figure 9 compares 1-np designs based on prototypes in the training data (selection, (S) to those based on prototypes built from the training data (replacement, (B)). A replacement method (LVQTC) assumes the minimum for the average ratio of $\left|\mathbf{V}_{i}\right| /\left|X_{\mathrm{tr}, i}\right|$, while a selection method [BTS(3)] yields, albeit slightly, the smallest average test error rate. The "cluster of 6 " methods (shown within the dashed boundary) closest to the origin in Figure 9 contains three from each category, implying that it is equally likely that you can find a decent set of prototypes for 1-np classifier design using either strategy.

Figure 10 compares pre- to postsupervision. The "cluster of 6" in Figure 10 contains five $\mathrm{P}$ s and only one (p): this seems to confirm what your intuition suspects, that using the labels to derive $\mathbf{V}$ is usually more effective than using them to label $\mathbf{V}$ after finding it. On the other hand, the two presupervised "outlier" designs in Figure $10(\mathrm{~W}+\mathrm{H}, \mathrm{GA})$ are all much worse than the three postsupervised methods (HCM, VQ, GLVQ-F), so it is not the case that presupervision is always better than postsupervision. Do not forget that Figure 10 is based on computations with four pretty small data sets, and we would not be too shocked if you found four other data sets where the conclusions suggested by your calculations were very different than these. 


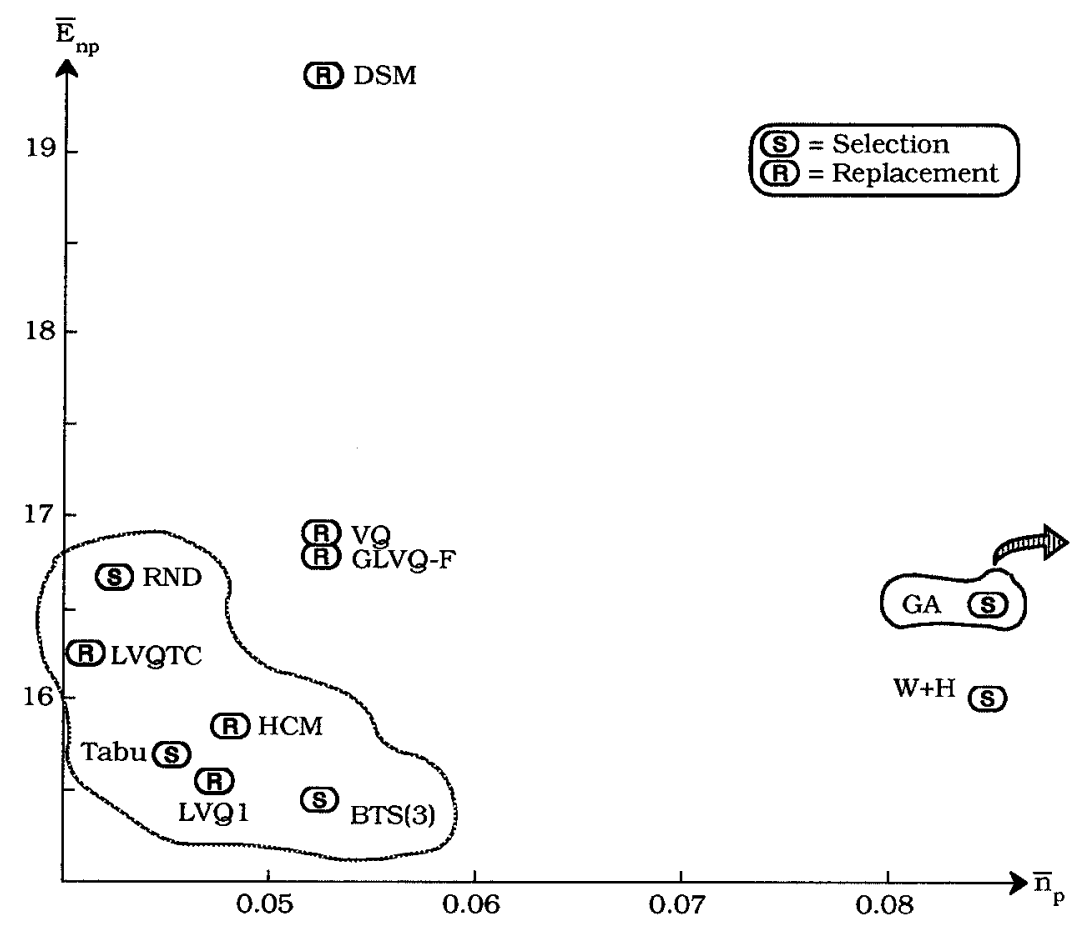

Figure 9. Selection vs. replacement: averages over four data sets.

Figure 11 compares methods that find the number of prototypes automatically (Auto- $n_{p}$, (A)) to methods in which the user has to tell the algorithm how many to look for (user- $n_{p}$, (U)). Thus, we see that the minimum number of prototypes is discovered automatically by LVQTC, while the minimum average error rate is produced by BTS(3), a method in which the user selects the number of prototypes, presumably with trial and error comparisons. Figure 11 does not provide conclusive evidence for either style of prototype generation, since the "cluster of 6 " in Figure 11 contains three auto- $n_{p}$ and three user- $n_{p}$ methods.

Figure 12 combines Figures 9 through 11 on one graph, where each of the ten plotted 1-np classifiers is associated with one of the three coordinate triples $(\mathrm{C} 1, \mathrm{C} 2, \mathrm{C} 3)$ from Table I. As in Figures 5 through 11, the "best" designs are the ones closest to the origin, and the four Pareto-optimal designs ${ }^{36}$ for averages over the four data sets are captured by the shaded region in Figure 12. The coordinates of these four designs in $(\mathrm{C} 1, \mathrm{C} 2, \mathrm{C} 3)$ space show ratios of: $3: 1$ for replacement vs. selection; 4:0 for presupervised designs vs. postsupervised designs; and 2:2 for auto- $n_{p}$ vs. user- $n_{p}$ selection of the number of prototypes. This indicates that - at least for these data sets and trials - presupervised, replacement prototypes are the more desirable combination of $(\mathrm{C} 1)$ and $(\mathrm{C} 2)$, while finding the best number of prototypes is done equally well by user specification or "automatically." 


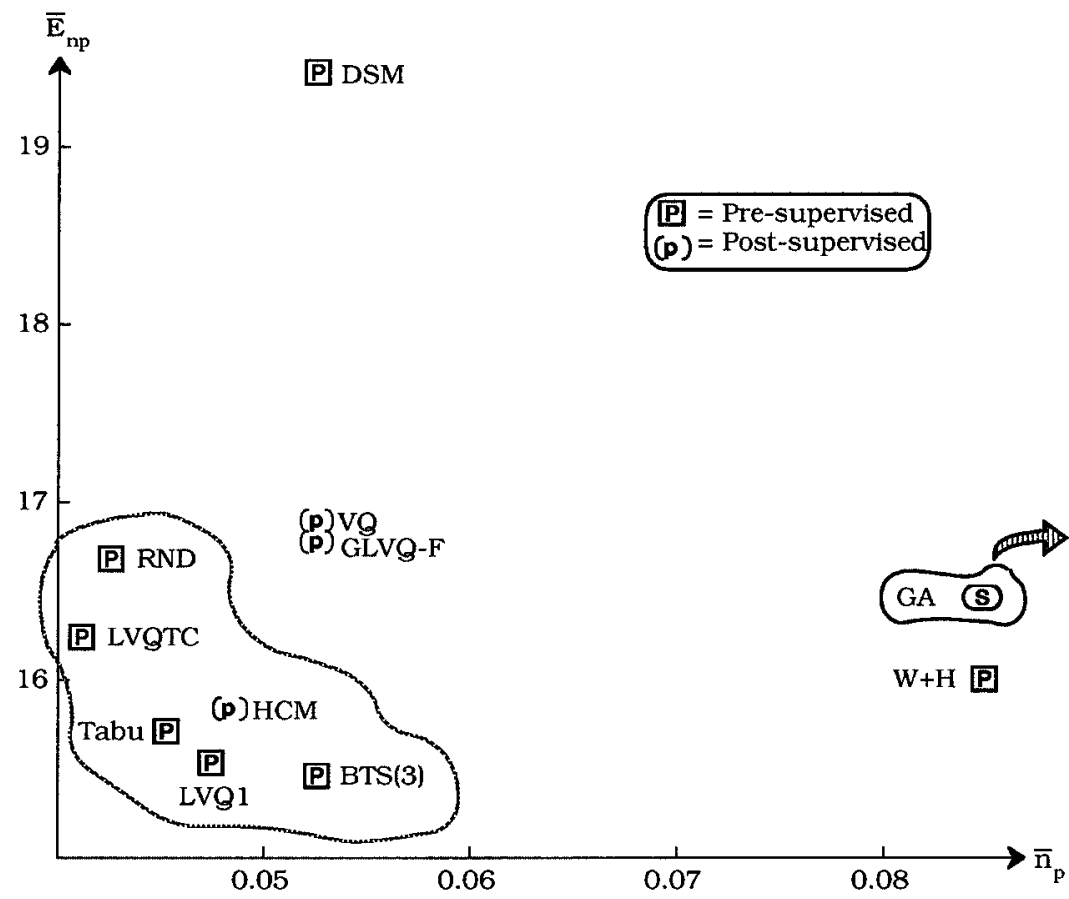

Figure 10. Presupervised versus postsupervised designs: averages over four data sets.

\section{CONCLUSIONS}

We discussed 11 methods for 1-np classifier design, all of which fall under the umbrella of the generalized nearest prototype classifier model. ${ }^{5}$ Our study explored the interaction of three characteristics of 1-np designs: (C1) Selection $\left(\mathbf{V}_{s} \subseteq X\right)$ versus replacement $\left(\mathbf{V}_{r} \not \subset X\right)$; (C2) Presupervised versus postsupervised designs; and (C3) User-defined $n_{p}$ (user- $\left.n_{p}\right)$ versus algorithmically defined $n_{p}$ (auto- $n_{p}$ ). The error rate on test data and the cardinality of the prototype set were the two criteria for comparison. Not surprisingly, different designs were better for different data sets (see Figs. 5 through 8). Moreover, no clear tendency emerged with respect to (C1), (C2), or (C3). Overall, Tabu search and LVQTC appeared to be the most successful designs. We believe that part of the success of Tabu search is due to the "constructive" initialization scheme of Cerveron and Ferri. ${ }^{16}$ In our experiments Tabu search found, usually within the first 20 iterations, an excellent initial guess which was almost never changed later in the algorithm, when the "do-not-delete" restriction is not in force. However, our study did not attempt to find a "best" 1-np design, but rather, to explore the importance of the three characteristics of all 11 designs. What can we say about each of these?

Our general belief about (C1) is that $\mathbf{V}_{r} \not \subset X$ can be better positioned (for 1-np classification) but more difficult to find than $\mathbf{V}_{s} \subseteq X$. Figure 12 shows that 


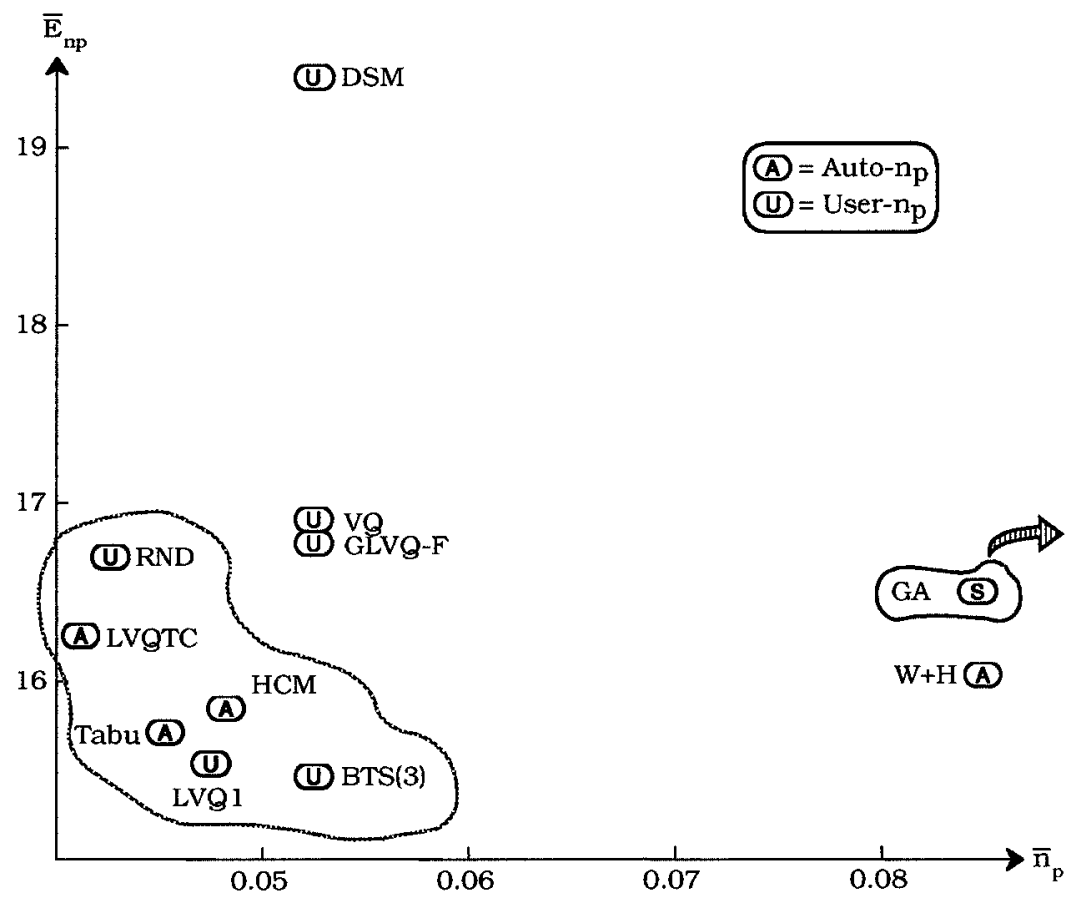

Figure 11. User-p vs. Auto-p: averages over four data sets.

on average over four sets of data, three of the four best methods are replacement methods. These three methods [LVQ1, LVQTC, BTS(3)] share the property that they build replacement prototypes by optimizing local criteria instead of a global criterion such as that used by batch algorithms like HCM.

For (C2), intuition suggests that presupervised methods are better than postsupervised schemes because they use the additional information possessed by the labels of the training data used to find $\mathbf{V}$. Figures 11 and 12 make it clear that presupervised methods were superior for these four data sets and 11 methods. This finding is consistent with that of an earlier study, where all five Pareto-optimal designs among 16 1-np classifiers that were compared with resubstitution errors committed in the Iris data were presupervised. ${ }^{7}$ We are confident enough about this point to assert that some presupervised method will almost always produce labeled prototypes for 1-np classifier design that are superior to those found by postsupervised methods. You noticed our use of the words "some" and "almost always"? Some, because in pattern recognition the trick is always to find the right classifier; almost always, because there is always a data set out there which provides a glaring counterexample to anything you claim is always true. For an example, just notice that DSM, W $+\mathrm{H}$, and GA (which falls outside the frame in Fig. 12) are also presupervised designs, but they were all inferior to all of the postsupervised designs. 


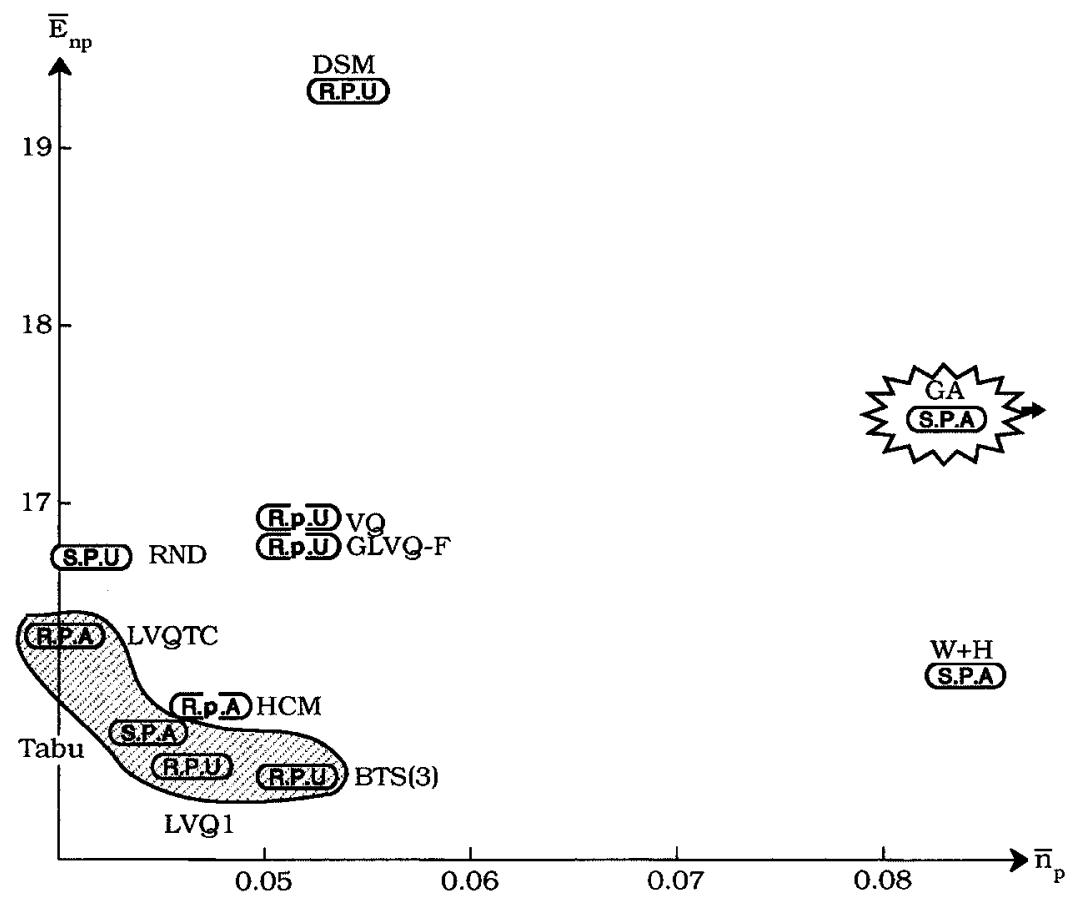

Figure 12. (C1, C2, C3) triples: averages over four data sets.

For (C3), auto- $n_{p}$ methods will certainly be more popular than user- $n_{p}$ designs, because they seem simpler to use. However, auto- $n_{p}$ methods simply hide the trial and error procedure for finding the "best" number of prototypes from the user, and instead rely on some mathematical criterion that selects $n_{p}$. If the criterion is test error $E_{\mathrm{np}}$, this relieves the user from conducting the trials and committing the errors (but necessitates the use of a validation test set; otherwise, there is no reason to believe that auto- $n_{p}$ methods will be superior to user- $n_{p}$ designs, and Figure 12 confirms this.

For each of the four data sets there is a fairly large group of designs with similar performance (those near the origin in Figures 5 through 12). The difference in testing errors or in the number of prototypes retained is small, and from our viewpoint these designs are all more or less equivalent. The GA design is missing from Figures 5 through 12 because the number of prototypes found by it was so high that if we put GA on the scatterplot, the scale would obscure differences between some of the other designs. In our previous study (Kuncheva and Bezdek ${ }^{6}$ ), GAs exhibited very good performance. Do we have an explanation for this inconsistency with earlier performance? Nope. Would other choices for $\alpha, p s, T$, the initialization and mutation probabilities, and the fitness function lead to better results? Sure-but then, you can improve any of these designs if you have enough time and patience. 
What can we conclude about the tradeoff between test error rate and the number of prototypes used? Nothing new, but these new experiments do support our convictions about this point more strongly. This tradeoff exists, and the general trend you would expect- that plots of $n_{p}$ vs. $E_{\text {np }}$ take the general shape of the graph of $y=1 / x$-holds throughout Figures 5 through 12. Perhaps the most interesting thing we can observe about this aspect of the experiments is that none of the auto- $n_{p}$ methods decided that $n_{p}$ as small as $c$, or $n_{p}$ as large as $\left|X_{\mathrm{tr}}\right|$, provided the best solution. From this we conclude that you will almost always do better with $n_{p}>c$, i.e., more prototypes than there are classes in the training data, but should almost certainly stop short of using as many prototypes as there are training data (in this latter case, if the prototypes are being selected, you arrive at the $k$-nearest neighbor rule, which is probably a better choice than 1-np classifiers anyway-but that is another story).

\section{References}

1. Bezdek JC, Keller J, Krishnapuram R, Pal NR. Fuzzy models and algorithms for pattern recognition and image processing. Norwell, MA: Kluwer; 1999.

2. Domingos P. Context-sensitive feature selection for lazy learners. Artificial Intelligence Review 1997;11:227-235.

3. Krishnapuram R, Keller JM. A possibilistic approach to clustering. IEEE Trans Fuzzy Systems 1998;1(2):98-110.

4. Zurada J. Introduction to artificial neural systems. St. Paul, MN: West Publ. Co.; 1992.

5. Kuncheva LI, Bezdek JC. An integrated framework for generalized nearest prototype classifier design. Int J Uncertainty, Fuzziness Knowledge-based Systems 1998; 6(5):437-457.

6. Kuncheva LI, Bezdek JC. On prototype selection: Genetic algorithms or random search? IEEE Trans Systems, Man, and Cybernet 1998;C28(1):160-164.

7. Kuncheva LI, Bezdek JC. Pre-supervised and post-supervised prototype classifier design. IEEE Trans Neural Networks 1999;10(5):1142-1152.

8. Kohonen T. Improved versions of learning vector quantization. Proc Int Joint Conf Neural Networks, San Diego, CA, 1990, p I-545-550.

9. Kohonen T. Self-organizing maps. Germany: Springer; 1995.

10. Diamantini C, Spalvieri A. Quantizing for minimum average misclassification risk. IEEE Trans Neural Networks 1998;9(1):174-182.

11. Diamantini C, Spalvieri A. Certain facts about Kohnonen's LVQ1 algorithm. IEEE Trans CAS-1: Fundamental Theory and Applications 1996;43(5):425-427.

12. Odorico R. Learning vector quantization with training counters (LVQTC). Neural Networks 1997;10(6):1083-1088.

13. Geva S, Sitte J. Adaptive nearest neighbor pattern classifier. IEEE Trans Neural Networks 1991;2(2):318-322.

14. Hart PE. The condensed nearest neighbor rule. IEEE Trans Inform Theory 1968;IT14:515-516.

15. Dasarathy BV. Nearest neighbor (NN) norms: NN pattern classification techniques. Los Alamitos, CA: IEEE Computer Society Press; 1991.

16. Cerveron V, Ferri FJ. Another move towards the minimum consistent subset: A tabu search approach to the condensed nearest neighbor rule (submitted).

17. Dasarathy BV. Minimal consistent set (MCS) identification for optimal nearest neighbor decision systems design. IEEE Trans Systems Man Cybernet 1994;24: 511-517. 
18. Wilson DL. Asymptotic properties of nearest neighbor rules using edited data. IEEE Trans Systems Man Cybernet 1972;SMC-2:408-421.

19. Devijver PA, Kittler J. On the edited nearest neighbor rule. In: Proc 5th Int Conf on Pattern Recognition. Los Alamitos, CA: IEEE Computer Society Press; 1980. p 72-80.

20. Devijver PA, Kittler J. Pattern recognition: A statistical approach. Englewood Cliffs, NJ: Prentice-Hall, Inc.; 1982.

21. Ferri FJ. Combining adaptive vector quantization and prototype selection techniques to improve nearest neighbor classifiers. Kybernetika 1998;34(4):405-410.

22. Skalak DB. Prototype and feature selection by sampling and random mutation hill climbing algorithms. In: Proc 11th Int Conf on Machine Learning, New Brunswick, NJ. Los Alamitos, CA: Morgan Kaufmann; 1994. p 293-301.

23. Chang EI, Lippmann RP. Using genetic algorithms to improve pattern classification performance. In: Lippmann RP, Moody JE, Touretzky DS, editors. Advances in neural information processing systems 3. San Mateo, CA: Morgan Kaufmann; 1991. p 797-803.

24. Kuncheva LI. Editing for the $k$-nearest neighbors rule by a genetic algorithm. Pattern Recognition Letters, Special Issue on Genetic Algorithms 1995;16:809-814.

25. Kuncheva LI. Fitness functions in editing $k$-NN reference set by genetic algorithms. Pattern Recognition 1997;30:1041-1049.

26. MacQueen J. Some methods for classification and analysis of multivariate observations. In: LeCam LM, Neyman J, editors. Proc Berkeley Symp Math Stat and Prob 1. Berkeley: Univ of California Press; 281-297.

27. Hamamoto Y, Uchimura S, Tomita S. A bootstrap technique for nearest neighbor classifier design. IEEE Trans Pattern Analysis and Machine Intelligence 1997; 19(1):73-79.

28. Xie Q, Laszlo CA, Ward RK. Vector quantization technique for nonparametric classifier design. IEEE Trans on Pattern Analysis and Machine Intelligence 1993; 15(12):1326-1330.

29. Karayiannis NB, Bezdek JC, Pal NR, Hathaway RJ, Pai P-I. Repairs of GLVQ: A new family of competitive learning schemes. IEEE Trans Neural Networks 1996; 7:1062-1071.

30. Bezdek JC, Reichherzer TR, Lim GS, Attikiouzel Y. Multiple prototype classifier design. IEEE Trans Systems Man Cybernet 1998;C28(1):67-79.

31. Duin RPW. PRTOOLS, Version 2. A Matlab toolbox for pattern recognition. Pattern Recognition Group, Delft University of Technology, June, 1997.

32. Ripley BD. Pattern recognition and neural networks. Cambridge, UK: University Press; 1996.

33. Holmstrom L, Koistinen P, Laaksonen J, Oja E. Neural and statistical classifiers-taxonomy and two case studies. IEEE Trans Neural Networks 1997; 8(1):5-17.

34. Duda RO, Hart PE. Pattern classification and scene analysis. New York: John Wiley \& Sons; 1973.

35. Anderson JA. Logistic discrimination. In: Krishnaiah PR, Kanal LN, editors. Classification, pattern recognition of dimensionality, Handbook of Statistics Series, 2. North Holland; 1982. p 169-191.

36. Winston WL. Operations research, applications and algorithms, 3rd ed. Belmont, CA: Duxbury Press; 1994. 